\title{
Identification and profiling of high temperature responsive miRNAs in French bean (Phaseolus vulgaris L)
}

\author{
M. N. Jyothi ${ }^{1,3}$, S. Usha ${ }^{1,3}$, B. Suchithra ${ }^{1,4}$, N. Sharadamma ${ }^{2}$, D. V. Rai ${ }^{3}$, V. R. Devaraj ${ }^{4}$, R. Nagesh Babu ${ }^{*}$ \\ ${ }^{1}$ Post Graduate Department of Biochemistry, Maharani's Science College for Women, Bangalore-560001. ${ }^{2}$ Department of Biochemistry, Indian Institute of \\ Science, Bangalore -560012 India. ${ }^{3}$ Centre for Bioinformatics, Faculty of Biological Engineering, Shobhit University, Meerut, India. ${ }^{4}$ Department of \\ Biochemistry, Central College Campus, Bangalore University, Bangalore -560001 India.
}

\section{ARTICLE INFO}

Article history:

Received on: 02/09/2016

Revised on: $24 / 09 / 2016$

Accepted on: 18/10/2016

Available online: $05 / 11 / 2016$

Key words:

miRNA; MYB; quantitative

PCR; Transcription factors.

\begin{abstract}
MicroRNAs (miRNAs) are group of small, non-coding RNAs that play important roles in plant growth, development and stress response. There have been an increasing number of investigations aimed at discovering miRNAs and analyzing their functions in model plants. In this study, we constructed high temperature stress induced small RNA libraries and characterized 26 potential miRNAs belonging to 21 families in French bean. A total of 140 annotated potential targets were found, of which majority were transcription factors (MYB, $b H L H$, GRF1, bZIP, NAC etc.,) which may play an important role in stress resistance. RT-qPCR and Northern blot analysis revealed differential expressions of candidate miRNAs and their target genes. The observed induction of miRNA expression is correlated with the down regulation of their targets. Investigation of gene ontology linked with targets of miRNAs forecasted their involvement in various biological functions. We anticipate the further studies may offer new avenues in developing stress tolerant variety of French bean.
\end{abstract}

\section{INTRODUCTION}

The modern globalization and extensive industrialization has resulted in reduced agricultural production. The exposure to various stressful environmental conditions and their sessile nature made plants to evolve advanced adaptive strategies to cope and sustain. These adaptations exhibited as physiological responses which were fine-tuned through induction of cascades of molecular mechanisms operating at transcriptional levels [1]. Advancement in the genomic technologies emphasized the role of non-coding RNAs as king-tuners of genomes and un-wrapped their involvement in genome dynamics, evolution and regulation. miRNAs are 21-24 nucleotide long, single stranded RNA molecules coded by their own transcripts known to exhibit gene regulation through target mRNA cleavage or translational repression mediated via RISC complex. Several roles of gene

* Corresponding Author

Dr. Nagesh babu R, Post Graduate Department of Biochemistry, Maharani's Science College for Women, Palace Road, Bangalore-560001

India.Email: nageshbabur@gmail.com;Ph: +91 22262796/09739438698 Fax: 08022342438 regulation have been attributed to these molecules which include development, signalling, defence and stress response. A number of miRNAs were demonstrated to function in biotic and abiotic stress responses in plants [2-4]. The role of miRNAs in plants infected by pathogenic bacteria, viruses, nematodes, and fungi has been widely reported [5, 6]. Various functions of miRNAs during abiotic stress has been established, which include cold [2, 7], drought [8] and oxidative stress induced by heavy metals, salinity, and nutrient deficiency [9]. Diverse set of miRNAs were also identified with heat stress response in wheat [10], Brassica rapa [11], barley [12]. French bean is one of the most important legume crop grown worldwide.

The major cues affecting the crop yield includes high temperature, nutrient deficiency, drought and high salinity. Constant rise in ambient temperature has been considered as the detrimental factor and affect the life processes which are multifarious, often lethal bringing alterations in plant growth, development, physiological processes and yield. Majority of the studies have concentrated on regulation of genes which are responsible for synthesis of osmo-protectants, detoxifying enzymes, transporter and other regulatory proteins [1]. 
Recently Arenas-Huertero et al. [13] identified conserved and novel miRNAs which responded to drought and salinity. In addition Valdes-Lopez et al. [14], used macroarray-hybridization approach to identify miRNAs responded to nutrient deficiency and manganese toxicity and demonstrated the involvement of miR399 in the PHR1 signaling pathway. Recently, Pelaez et al. [15] used high throughput sequencing for the identification and characterization of $P$. vulgaris miRNAs under salt stress. Previously we have reported the altered expression of miRNAs render plant tolerant towards salt, drought and nutrition depletion $[16,17]$. In this study, we aimed to decipher a set of conserved and novel miRNAs responding to high temperature stress. The stress modulated expression of miRNAs was validated through stemloop quantitative PCR and Northern blot to establish the negative correlation of expression of the candidate miRNAs and their targets. The gene ontology studies revealed many of the targets identified with the candidate miRNAs were involved in maintaining cellular homeostasis.

\section{MATERIALS AND METHODS}

\subsection{Plant materials and stress treatment}

Seeds of French bean (Phaseolus vulgaris Selection - 9) were surface sterilized and grown under controlled conditions at $28{ }^{\circ} \mathrm{C}$ day $/ 25{ }^{\circ} \mathrm{C}$ night with $12 \mathrm{~h}$ light $/ 12 \mathrm{~h}$ dark photo period. After 6 day of germination, seedlings were exposed to high temperature stress $\left[42^{\circ} \mathrm{C}\right.$ for $1 \mathrm{~h}$ (induction); $45^{\circ} \mathrm{C}$ for $1 \mathrm{~h}$ and 48 ${ }^{\circ} \mathrm{C}$ for $6 \mathrm{~h}$ respectively]. Tissues were harvested immediately and stored at $-80{ }^{\circ} \mathrm{C}$ for further analysis.

\subsection{Small RNA extraction and cloning}

Total RNA was isolated from tissue using TRizol (Invitrogen) according to the manufacturer's instructions and treated with RNAase-free DNAase I (Promega). Small RNAs (200 nt) were separated on a denaturing $15 \%$ polyacrylamide gel. Molecules ranging from 18 to $26 \mathrm{nt}$ were excised and recovered. The sRNAs were then 3' (5'-ACTGTAGGCACCATCAAT-3' underlined BanI site) and (5'AAACCATGGTACTAATACGACTCACTAAA-3' underlined RsaI site) adapter-ligated by T4 RNA ligase (Fermentas). At each step, their lengths were verified and purified by Urea-PAGE. The adapter-ligated sRNAs were transcribed into cDNA by SuperScript II Reverse Transcriptase (Invitrogen). Reverse transcription was performed using the adapter primers and the resulting cDNAs were PCR amplified with family specific primers at $95^{\circ} \mathrm{C}$ for $20 \mathrm{~s}$; $56-58^{\circ} \mathrm{C}$ for $30 \mathrm{~s} ; 72^{\circ} \mathrm{C}$ for 30 s cycled for 30 and final extension at $72{ }^{\circ} \mathrm{C}$ for $5 \mathrm{~min}$ in thermo cycler (Kyratec). The amplicon were purified and ligated into $p G E M T$ Easy (Promega). The colonies were screened for the ligated products using gene specific primers. The plasmid DNA from recombinant colonies showing the correct product size were subjected to RsaI and BanI digestion and rechecked for the insert size. This procedure screened out the colonies carrying adapter self-ligation and possibly the degraded mRNA products carrying the restriction sites. The colonies carrying the correct size fragments were further screened using the adapter primers in combinations with vector primers. The PCR positive clones were sequenced and processed for BLAST analysis against the NCBI genomic data sets.This strategy also revealed that in some colonies two miRNAs were ligated together.

\subsection{Data analysis}

$\begin{array}{cccr}\text { The } & \text { sequences were } & \text { filtered for } & \text { tRNA/rRNA } \\ \text { contamination } & \text { using } & \text { Rfam } & \text { database }\end{array}$ (http://www.sanger.ac.uk/Software/Rfam). Putative origins for the remaining sequences were identified by BLASTN search against French bean genome sequence. The sequences with perfect (0-3) matches with small RNA sequences were used for fold back secondary structure prediction with MFOLD [18]. A segment was considered a valid miRNA candidate if its secondary structure met the criteria according to Meyers et al. [19]

\subsection{Prediction of Potential Target mRNAs}

Target prediction for the miRNAs was based on the principle of nearly perfect complementation between the miRNA and target mRNAs. The identified conserved and putative novel miRNAs were all submitted for target gene prediction using psRNATarget (http://plantgrn.noble.org/psRNATarget/) and psRobot (http://omicslab.genetics.ac.cn/psRobot/). French bean transcript sequences downloaded from Phytozome version 9 (www.phytozome.net ) were used to predict the putative targets with default parameters. Sequences with a score of less than 4 were regarded as miRNA target genes. On the basis of their functions putative targets were classified using Gene Ontology (GO) annotations from agriGO (http://bioinfo.cau.edu.cn/agriGO).

\subsection{Semi-quantitative RT-PCR validation of miRNA Expression}

To validate the expression of the high temperature stress miRNAs in French bean, stem loop RT-qPCR was performed using SYBR Green PCR Master mix (Takara) on Light cycler $96^{\circledR}$ (Roche). Each PCR reaction $(20 \mu \mathrm{l})$ included $2 \mu \mathrm{l}$ cDNA, 10 $\mu \mathrm{l}$ SYBR Green Master mix, $1 \mu \mathrm{l}$ sequence specific forward primer $(10 \mu \mathrm{M}), 1 \mu \mathrm{l}$ Universal reverse primer $(10 \mu \mathrm{M})$ and $6 \mu$ l sterile water. The miRNA expression was normalized against U6 gene. The reactions were performed at $95{ }^{\circ} \mathrm{C}$ for $10 \mathrm{~min}$ followed by 40 cycles of $95{ }^{\circ} \mathrm{C}$ for $15 \mathrm{~s}$ and $60{ }^{\circ} \mathrm{C}$ for $1 \mathrm{~min}$ with a final dissociation $72{ }^{\circ} \mathrm{C}$ for 30 s, with two biological replicates; the data was analysed based on efficiencies and fold changes. For target validation each reverse transcriptase reaction contain $2.5 \mu \mathrm{g}$ of total DNA free RNA, $1 \mu \mathrm{l}$ oligo dT and $1 \mu \mathrm{l}$ dNTPs mix $(10 \mu \mathrm{M})$. The reaction was incubated for $5 \mathrm{~min}$ at $65^{\circ} \mathrm{C}$ and snap cooled subsequently, 5X First strand buffer, DTT, Ribolock and Superscript II enzyme (Invitrogen) were added. This reaction was incubated for $30 \mathrm{~min}$ at $42{ }^{\circ} \mathrm{C}$ followed by $70^{\circ} \mathrm{C}$ using forward and reverse gene specific primers. The reactions were carried as described earlier and signals were quantified relative to GAPDH mRNA using the $2^{-\Delta \Delta \mathrm{CT}}$ method. 


\subsection{Northern Blot analysis}

In order to validate the miRNA expression, northern blot hybridization was conducted using high sensitive miRNA Northern blot assay kit (Signosis, USA). $30 \mu \mathrm{g}$ total RNA of each sample was electrophoresed on $15 \%$ polyacrylamide gel and transferred to membrane. Antisense RNA biotin labeled in the 5' end (Invitrogen) was used as hybridization probes. The SYBR Green ${ }^{\circledR}$ II stained (Biotech) 5S rRNA was used as loading control. All the primers used in the study are mentioned in Suppl. 1.

\section{RESULTS AND DISCUSSION}

\subsection{Identification of miRNAs and their targets}

To explore the role of miRNAs under high temperature, small RNA libraries were constructed from stress exposed seedlings. 3,000 clones were screened for the sequences representing candidate miRNAs. $80 \%$ of the sequences were 18-26 nt in length and the remaining sequences had either shorter fragments or self -ligated adapters. Further, the sequences were mapped to French bean nuclear genome (http://www.phytozome.net), 97\% of the sequences exhibited at least one match with the genome. Several sequences were mapped to chloroplast/mitochondrial genomes and few represent possible regulatory products/orgenellar RNAs (Figure 1). The tRNA/rRNA contamination was clarified by Blastn against Rfam database.
Based on initial survey of secondary structure and homology of mature miRNA sequences with miRBase v20 (www.miRBase.org), we were able to identify 26 conserved miRNAs corresponding to 21 miRNA families and two miR* sequences with two predominant size classes of 20 and $21 \mathrm{nt}$ (Figure 2).

The detection of miRNAs is a strong clue, albeit not infallible, about the existence of precursor hairpin structures with negative folding-free energies ranging from -19.99 to $-82.28 \mathrm{~kJ}$ and their MFEI was <- 0.85 adds credence to the authenticity of the predicted candidates (Table 1). Majority of the sequences are starting with 5' Uridine, which is in accordance with defined structure of miRNAs. The miRNA sequence that remained after exclusion of conserved and their variants were considered as novel. These sequences exhibited $\geq 4$ mismatch with conserved miRNAs and has been named as $P v u N x$ (for Phaseolus vulgaris Novel, $\mathrm{x}$ being the number). We were able to identify 8 new members belonging to four miRNA families (Suppl. 2, Suppl. 3, and Suppl. 4).

To clarify the biological functions of the newly identified miRNAs, we examined putative target genes using the psRNATarget and psRobot programs with default parameters. We observed the candidate miRNA targeting many genes involved in different cellular functions suggests that these miRNAs may play multiple roles.

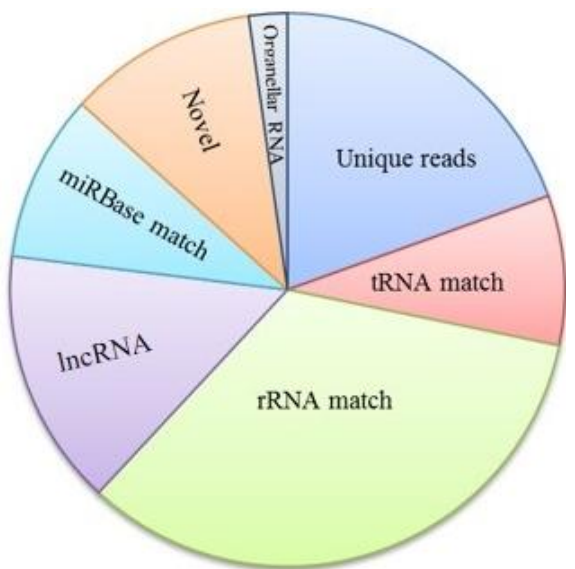

Fig. 1: Familial classification of small RNA raw sequences obtained from Sanger sequencing. The unique reads represent the small RNA reads filtered from Rfam. Novel miRNAs represent small subset of unique reads which does not show homology with conserved miRNAs from miRBase.

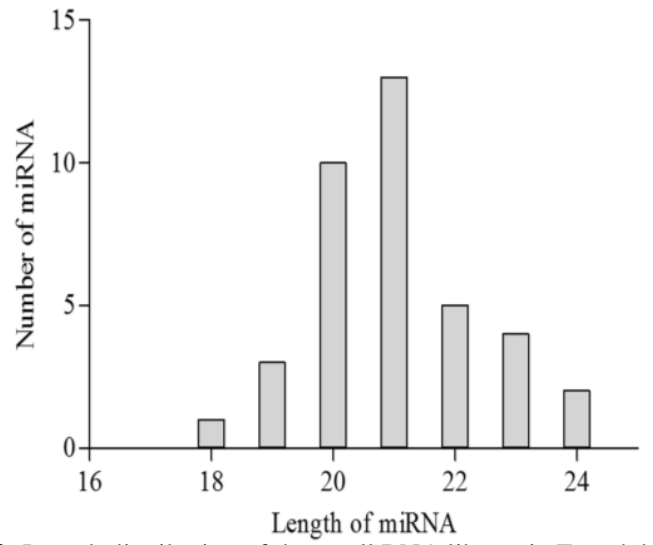

Fig. 2: Length distribution of the small RNA library in French bean. 
Table 1: Conserved miRNAs identified with French bean expressed under high temperature stress.

\begin{tabular}{|c|c|c|c|c|}
\hline miR & $\begin{array}{c}\text { Sequence } \\
\end{array}$ & Length & MFE & Family \\
\hline Pvu seq 3 & UGAAGUGUUUGGGGGAACUC & 20 & -80.3 & miR395 \\
\hline Pvu seq 5 & ACCAUCACCGAGGUGUACGA & 20 & -54.14 & $\operatorname{miR} 2111$ \\
\hline Pvu seq 6 & UAUGGCCGCUGCUGUGAUGAUGA & 23 & -13.85 & miR397 \\
\hline Pvu seq 7 & UAUGGCCGCUGCUGUGAUGAUGAUGA & 24 & -29.88 & $\operatorname{miR} 2653$ \\
\hline Pvu seq 8 & GUGGAGGUCGGUGCCGCG & 20 & -58.14 & $\operatorname{miR} 858$ \\
\hline Pvu seq 9 & GAUCAAAAUUUUAUCAUUUUUAC & 23 & -19.99 & $\operatorname{miR} 1438$ \\
\hline Pvu seq 10 & AUUCGUGUUCAGAAAGGAGA & 20 & -21.8 & $\operatorname{miR} 2674$ \\
\hline Pvu seq 11 & AGGACUCGGCAUGGCUCCUCCCAC & 24 & -61.45 & $\operatorname{miR} 160 *$ \\
\hline Pvu seq 13 & UGGGAGGAGCCAACCCUAGUG & 21 & -42.8 & $\operatorname{miR} 396$ \\
\hline Pvu seq 14 & AAGGCACAGUCAAUUCCAGGUU & 21 & -37.23 & $\operatorname{miR} 2905$ \\
\hline Pvu seq 16 & UUAUGUGUAAAUGAAUGAAAU & 21 & -18.4 & $\operatorname{miR} 319$ \\
\hline Pvu seq 17 & UUGGACUGAAGGGACCUCCUU & 21 & -18.4 & $\operatorname{miR} 319$ \\
\hline Pvu seq 18 & UGAAGCUGCCAGCAUGAUCU & 20 & -64.8 & $\operatorname{miR} 167$ \\
\hline Pvu seq 19 & AAGCUGCCAGCAUGAUCUGA & 20 & -53.02 & $\operatorname{miR} 167$ \\
\hline Pvu seq 20 & UUGGGCAAAUCUCCUUUGGCA & 21 & -41.55 & miR399 \\
\hline Pvu seq 21 & UUGCCGAUUCCACCCAUUCCUAU & 23 & -56.00 & $\operatorname{miR} 2118$ \\
\hline Pvu seq 22 & UUAAUUGAGCCGCGUCAAUAUC & 22 & -64.5 & $\operatorname{miR} 171$ \\
\hline Pvu seq 23 & ACGAUGAUGAUGAGGAUGA & 19 & -51.76 & miR414 \\
\hline Pvu seq 26 & ACAGAUCAUGUGGCUGCUUCA & 21 & 33.9 & $\operatorname{miR} 167$ \\
\hline Pvu seq 27 & UAUUAAUCUUGAUGAUGCUGCA & 22 & -40.3 & $\operatorname{miR} 172$ \\
\hline Pvu seq 28 & AGAAUCUUGAUGAUGCUGCA & 20 & -40.3 & $\operatorname{miR} 172$ \\
\hline Pvu seq 29 & GGAAUAUUGAUGAUGCUGAU & 20 & -37.4 & $\operatorname{miR} 172$ \\
\hline Pvu seq 32 & AAGACAUCGCCAAGGAGACU & 20 & -45.7 & $\operatorname{miR} 169$ \\
\hline Pvu seq 33 & CUAUUAAUCUUGAUGAUC & 18 & -44.0 & $\operatorname{miR} 172$ \\
\hline Pvu seq 34 & UUAAUCUUGAUGAUGCUGC & 19 & -29.9 & $\operatorname{miR} 172$ \\
\hline Pvu seq 35 & UGGUGCACUUGAUGAUGCUG & 20 & -31.5 & $\operatorname{miR} 172$ \\
\hline Pvu seq 36 & UGUGUUCUCAGGUCACCCCUU & 20 & -37.6 & $\operatorname{miR} 398$ \\
\hline
\end{tabular}

Suppl. 1: List of primers used in the study

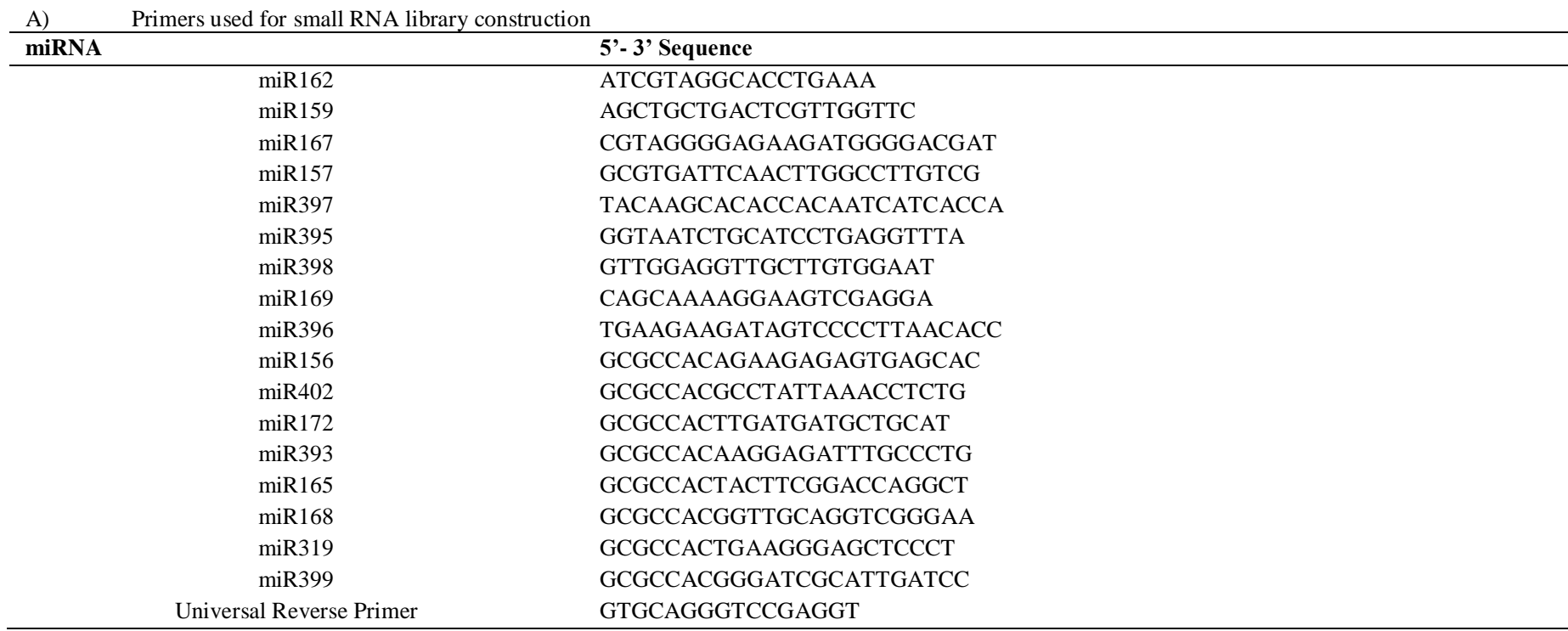

\begin{tabular}{cl} 
B) & Stem - loop primers used for mature miRNA validation \\
\hline miRNA & \multicolumn{1}{c}{ Primer sequence 5'-3' } \\
\hline Pv seq 3 & SL:GTCGTATCCAGTGCAGGGTCCGAGGTATTCGCACTGGATACGACGAGTTC \\
& FP:GCCGGGTGAAGTTGTTCC \\
& RP: CCAGTGCAGGGTCCGAGGTA \\
\hline Pv seq 10 & SL:GTCGTATCCAGTGCAGGGTCCGAGGTATTCGCACTGGATACGACTCTCCT \\
& FP:GCCGGGATTCGTGTTCAG \\
& RP: CCAGTGCAGGGTCCGAGGTA \\
\hline Pv seq 12 & SL: GTCGTATCCAGTGCAGGGTCCGAGGTATTCGCACTGGATACGAC FP:GATGCGACTCGGCATGGC \\
& RP: CCAGTGCAGGGTCCGAGGTA \\
\hline Pv seq 13 & SL:GTCGTATCCAGTGCAGGGTCCGAGGTATTCGCACTGGATACGACCACTAG FP: GATGCGTGGGAGGAGCCA \\
& RP: CCAGTGCAGGGTCCGAGGTA \\
\hline Pv seq 17 & SL:GTCGTATCCAGTGCAGGGTCCGAGGTATTCGCACTGGATACGACAAGGAG \\
& FP:GAGCTGGTTGGACTGAAGG RP: CCAGTGCAGGGTCCGAGGTA \\
\hline
\end{tabular}




\begin{tabular}{ll}
\hline Pv seq 20 & SL:GTCGTATCCAGTGCAGGGTCCGAGGTATTCGCACTGGATACGACTGCCAA \\
& FP:GCCGGGTTGGGCAAATCT \\
& RP: CCAGTGCAGGGTCCGAGGTA \\
\hline Pv seq 25 & SL:GTCGTATCCAGTGCAGGGTCCGAGGTATTCGCACTGGATACGACATACTC \\
& FP:GAGCTGGATATTGGGACGG RP: CCAGTGCAGGGTCCGAGGTA \\
\hline Pv seq 27 & SL:GTCGTATCCAGTGCAGGGTCCGAGGTATTCGCACTGGATACGACTGCAGC \\
& FP:GCGATGGCCTATTAATCTTGA \\
& RP: CCAGTGCAGGGTCCGAGGTA \\
\hline Pv seq 30 & SL:GTCGTATCCAGTGCAGGGTCCGAGGTATTCGCACTGGATACGAC \\
& FP:GAGCTGGCAATTTGGGTGC \\
& RP: CCAGTGCAGGGTCCGAGGTA \\
\hline Pv seq 36 & SL:GTCGTATCCAGTGCAGGGTCCGAGGTATTCGCACTGGATACGACAAGGGG \\
& FP:GGACGGTGTGTTCTCAGG \\
& RP: CCAGTGCAGGGTCCGAGGTA \\
\hline
\end{tabular}

\begin{tabular}{llll} 
C) & Primers used for expression analysis Target genes using qPCR & \\
\hline miRNA & Target gene & Expression & Primer sequence 5'-3' \\
\hline Pvu seq3 & ATP sulpharylase & Down regulated & $\begin{array}{l}\text { FP: GTAAGCTTGTTCCTCGGCCT } \\
\text { RP: GCATCAGCTTTGCATCTGCA }\end{array}$ \\
\hline Pvu seq10 & $\begin{array}{l}M Y B \text { - domain } \\
\text { protein }\end{array}$ & Up regulated & $\begin{array}{l}\text { FP: CTTCCTCTAGCTTTCTCTCGC } \\
\text { RP: AAATCGTAGTGGCGGTCTGA }\end{array}$ \\
& $\begin{array}{l}\text { Serine carboxy } \\
\text { peptidase }\end{array}$ & Down regulated & FP: AGTGAGCGAGTGAGTAGGGT \\
Pvu seq13 & Lipoyl transferase & Pown regulated & FP: TAAGAGCCCCTTGCAAGCTA \\
& RP: CGACCAAGGAAGTCCACAT \\
\hline Pvu seq16 & $\begin{array}{l}\text { Stress inducible } \\
\text { protein }\end{array}$ & Up regulated & FP: TCAATGATGGTTTGCCTCA \\
& Re: CATGAGCTTCTCGATGTCCA \\
\hline Pvu seq20 & Up regulated & $\begin{array}{l}\text { FP: TCTTCATGCTCAACTTGGCA } \\
\text { RP: CAGGTAGCTGCAGGTCTTGA }\end{array}$ \\
\hline Pvu seq36 21 & $\begin{array}{l}\text { Heat shock } \\
\text { transcription factor }\end{array}$ & Up regulated & FP: ACGAGATCGCTTCACACCTC \\
& & & RP: CAAAGGTACGCCGGGGATAA \\
\hline
\end{tabular}

Suppl. 2: Description of novel miRNAs characterized under high temperature stress.

\begin{tabular}{lllllll}
\hline miRNA & Sequence 5'-3' & Length & MFEI & Family & Chromosome no. & Genomic location \\
\hline Pvu N1 & CUUCAUCGAGGGGCUGUCUCC & 21 & -0.94 & miR1919 & Chromosome 11 & 3'UTR \\
Pvu N2 & UGCUGCCCCUCGGCGGUCUC & 20 & -0.98 & Undefined & Chromosome 02 & Intron \\
Pvu N3 & UUACCGGCGUUACGUGGAGUCG & 22 & -0.89 & Undefined & Chromosome 02 & 5' UTR \\
Pvu N4 & UUGCAGAACCUGGAAUUGACUGU & 23 & -0.81 & miR2905 & Chromosome 08 & CDS \\
Pvu N5 & AACAGGGCGGGGAACAGGUGGUG & 23 & -0.85 & miR2030 & Chromosome 01 & 3' UTR \\
Pvu N6 & AUAUUGGGACGGAGGGAGUAU & 21 & -0.84 & miR6224 & Chromosome 06 \& 08 & Intron \\
Pvu N7 & CAAUUUGGGUGCCCCUGCUG & 20 & -0.86 & Undefined & Chromosome 04 & 5'UTR \\
Pvu N8 & UUGGGUGCCCCUGCUGUUUCUU & 22 & -0.91 & Undefined & Chromosome 04 & 5'UTR \\
\hline
\end{tabular}

$M F E I=($ MFE/ Length of pri-miRNA $) * 100 \div G+C \%$

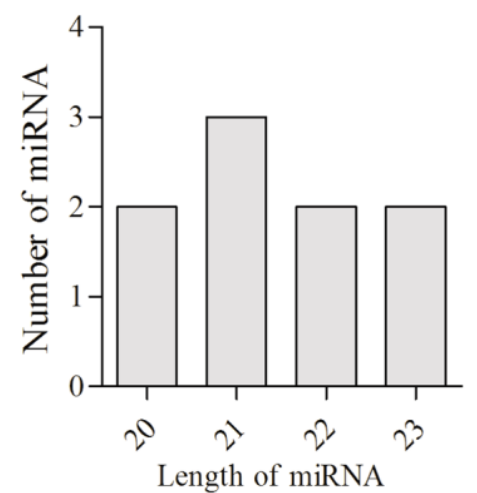

Suppl. 3: Length preference of novel miRNAs 
Suppl. 4: Structure of novel miRNAs

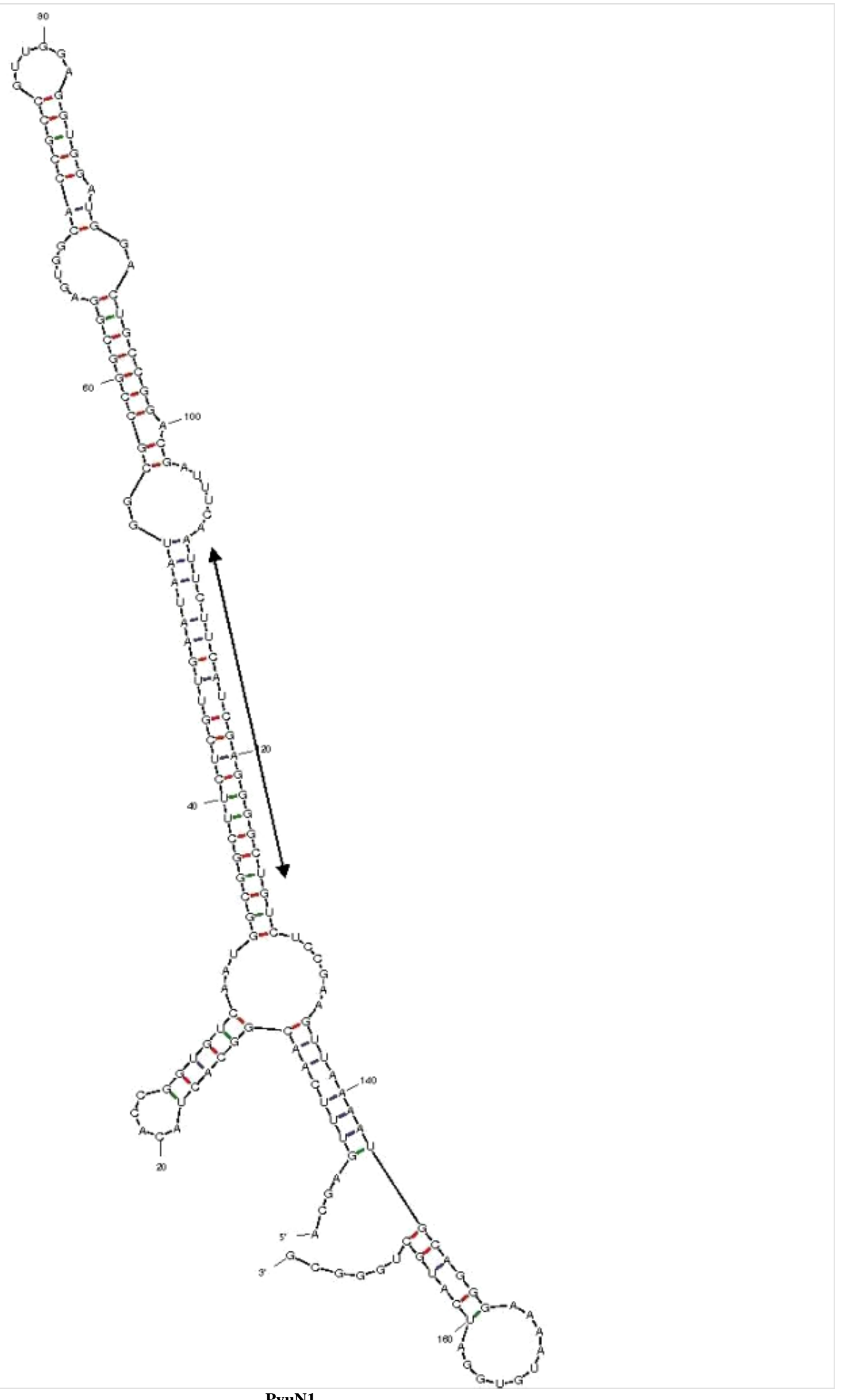




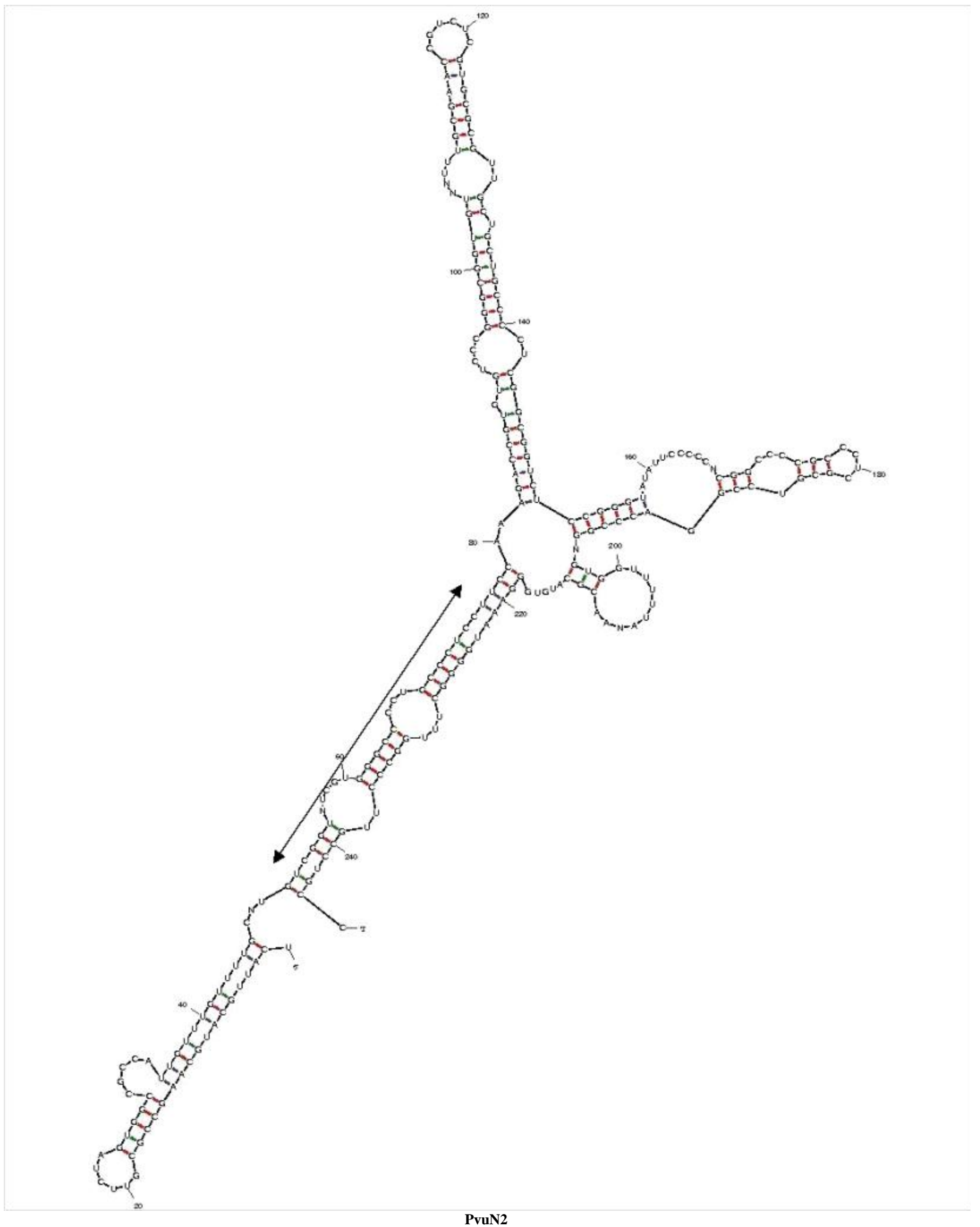




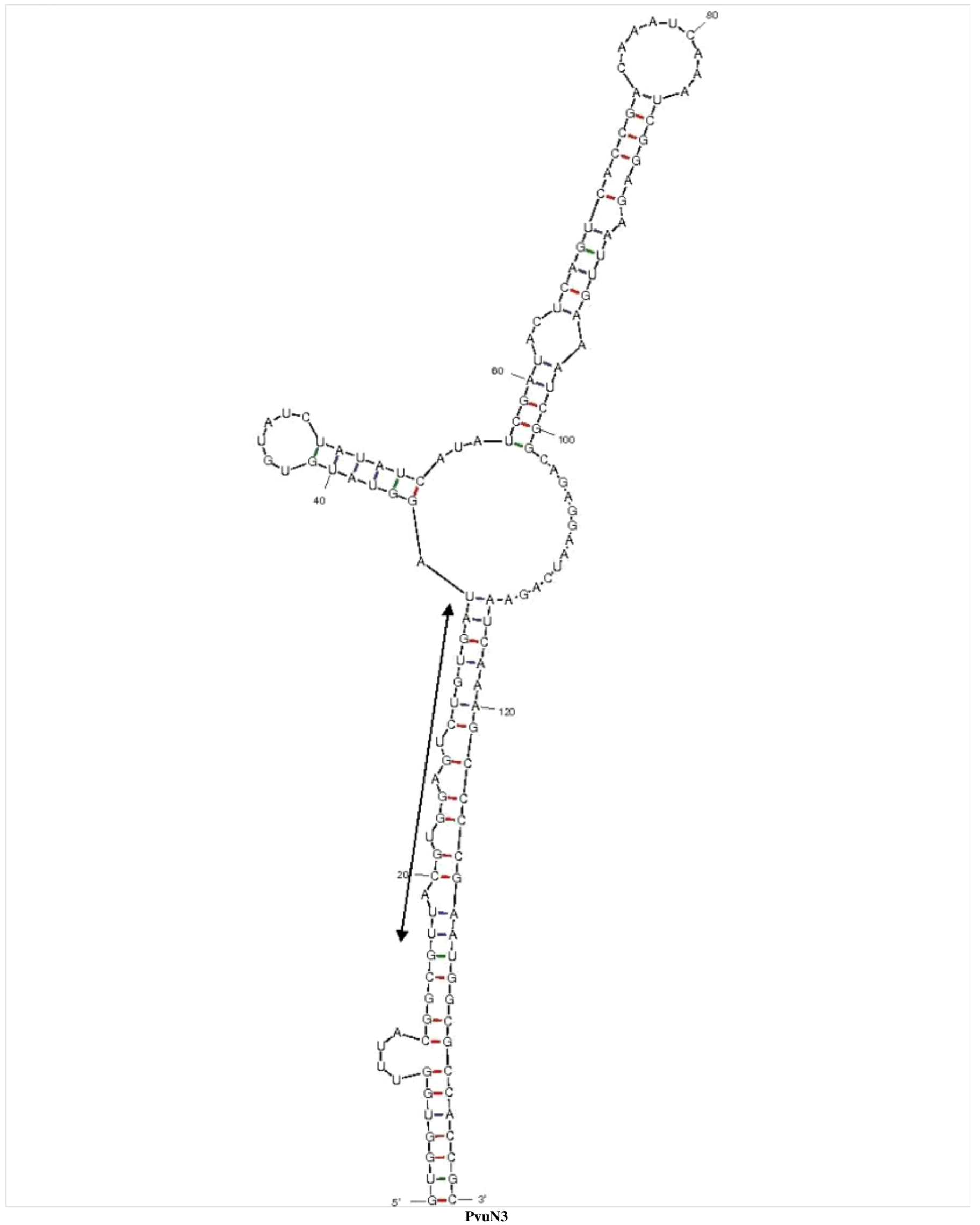




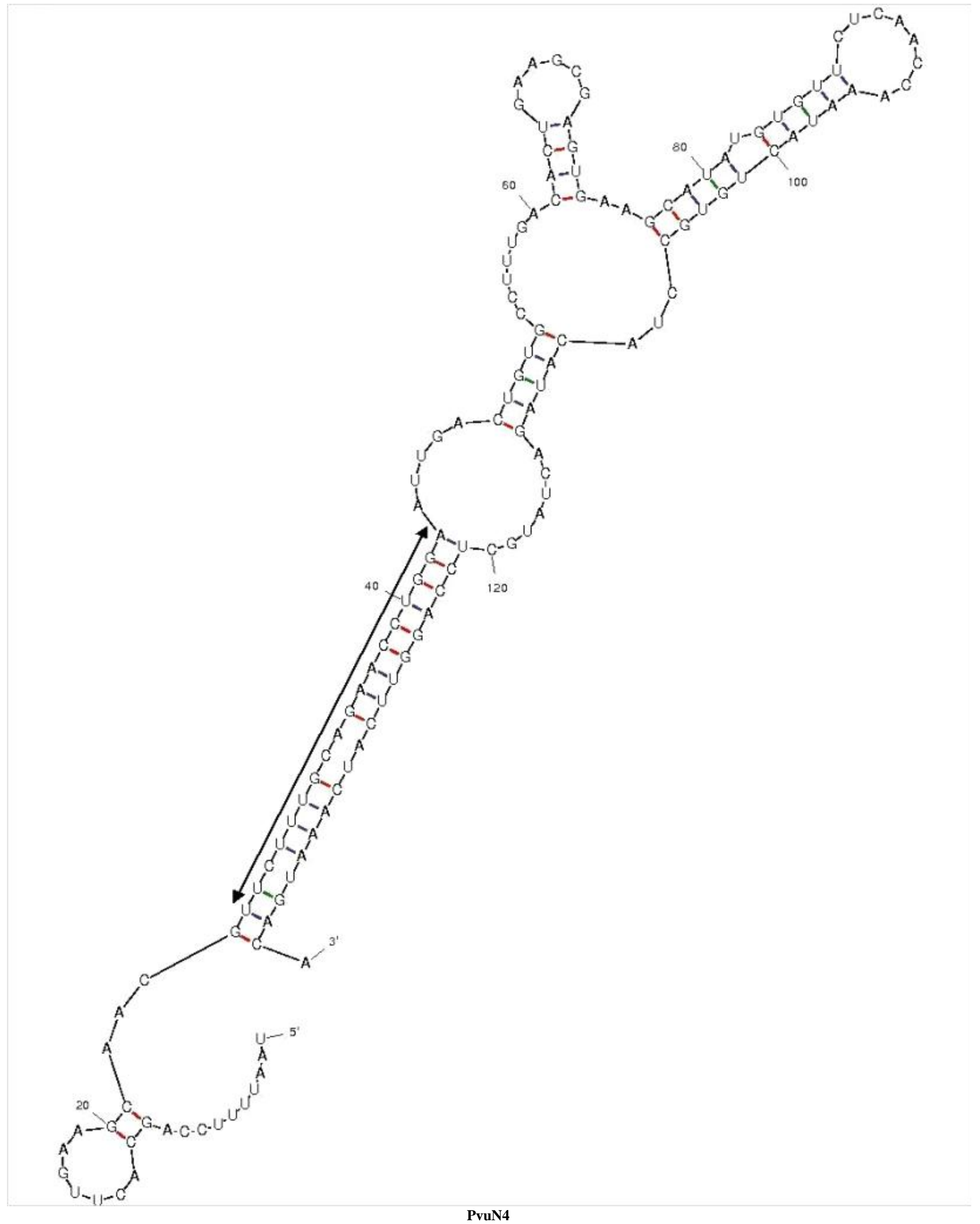




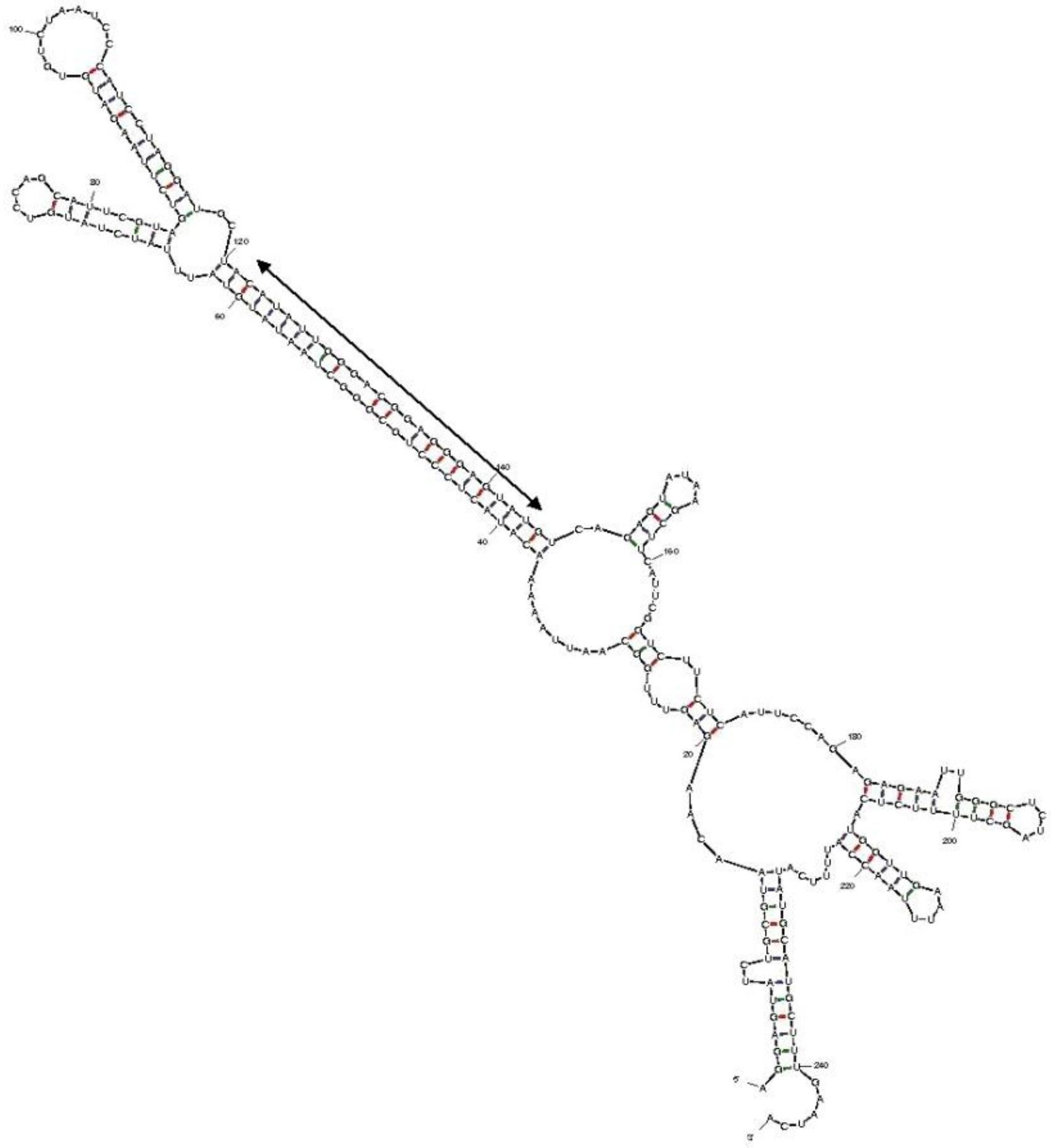

Pvun5 


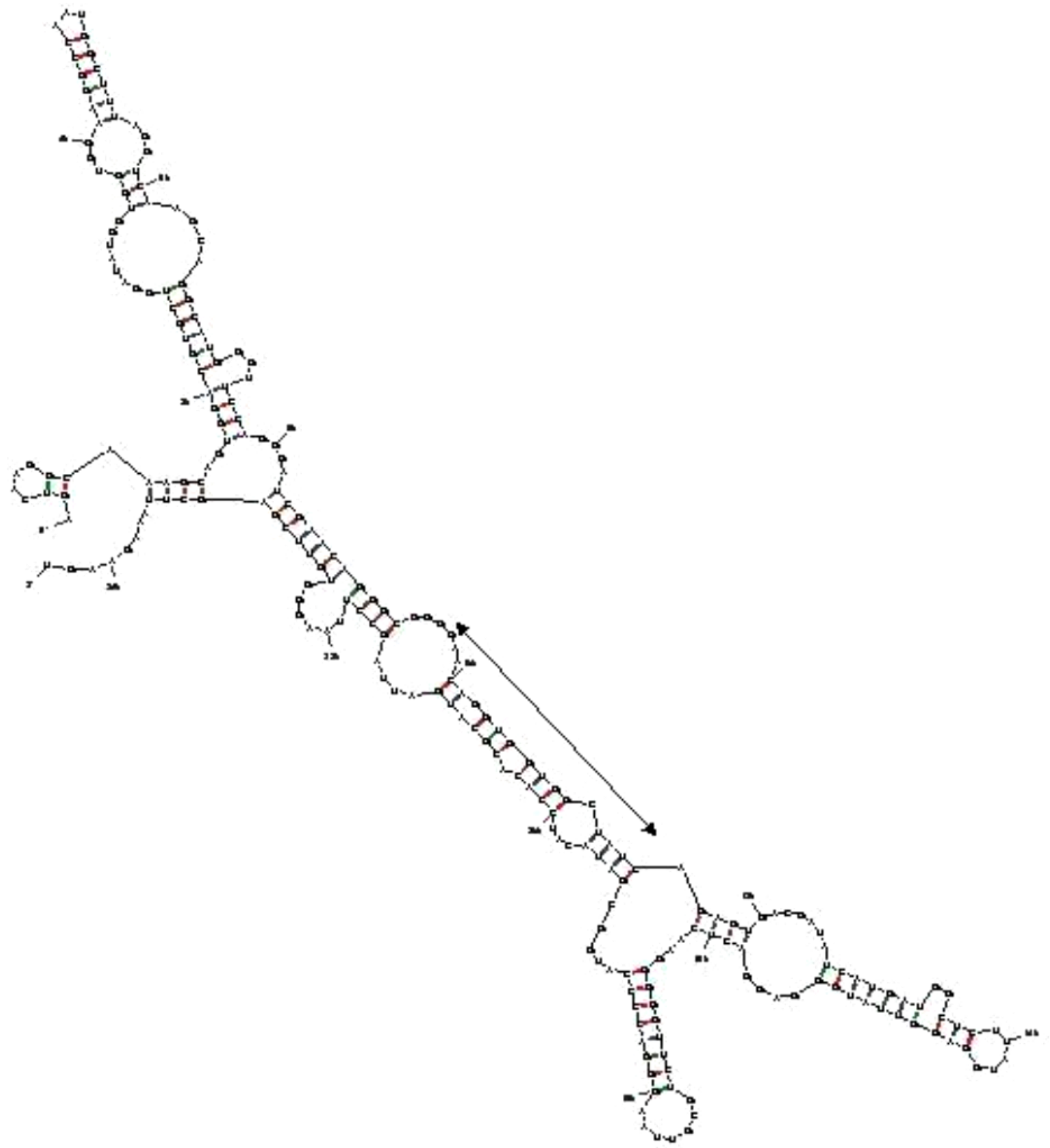

PvuN6 


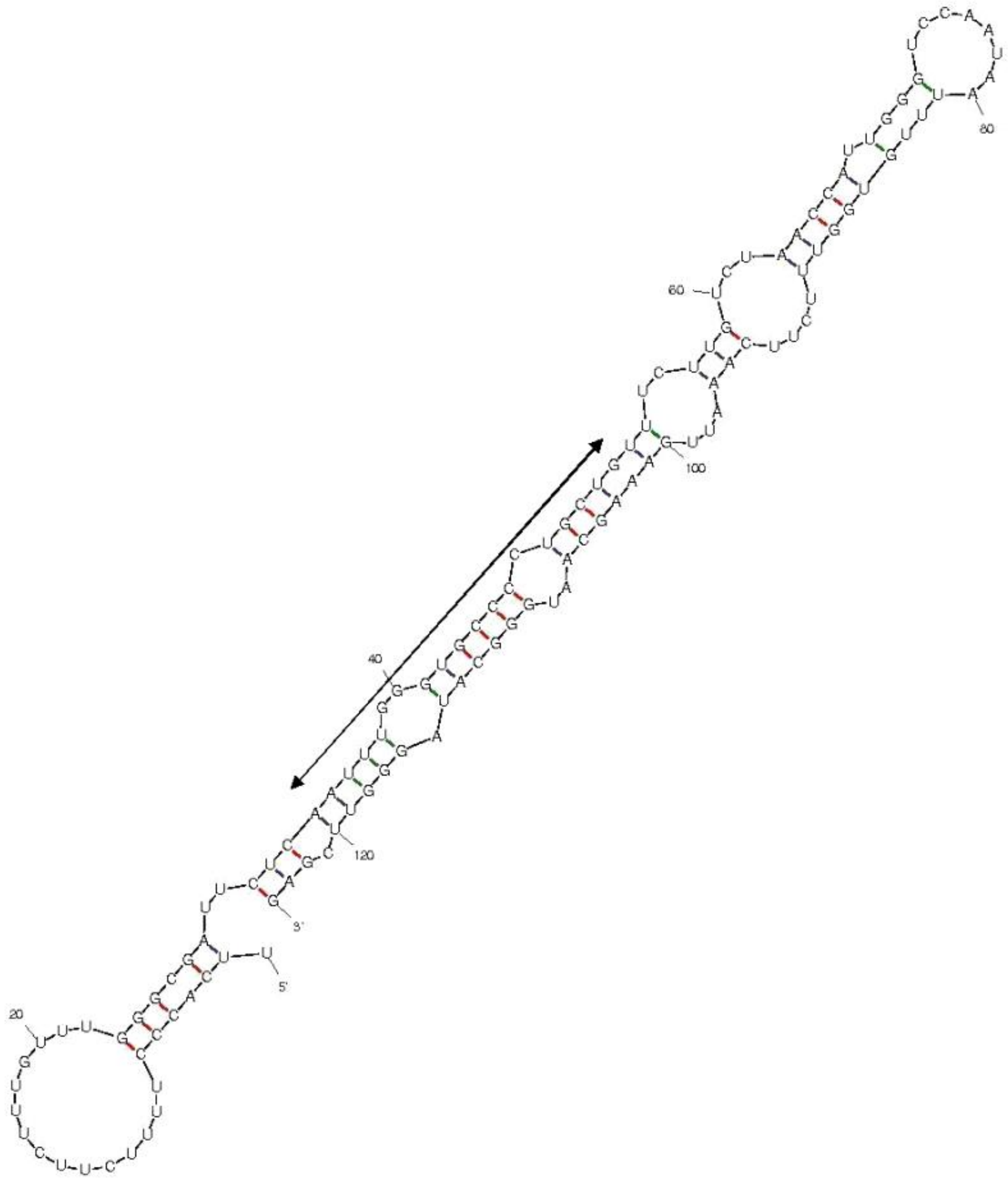


A total of 250 genes were analyzed; 140 genes were annotated and most of the miRNAs exhibited multiple targets. Pvu seq23 has maximum hits with 25 targets while, Pvu seq7, Pvu seq9, Pvu seq16, Pvu seq24, Pvu seq30, and Pvu seq32 targeted single gene. Recognition of multiple targets emphasizes the role of miRNAs in intricate gene regulatory network. Most targets were transcription factors which include NBS- LRR, MYB, bHLH, NAC, F-box, GRF1, bZIP, RING-H2 finger, AP2, ARF etc., others represent metabolic enzymes, membrane proteins and signal transducers (Table 2; Suppl. 5). To determine the roles of identified putative target annotation was performed. The frequency of GO terms associated with predicted targets were enriched with biological function and linked to molecular components (Figure 3). Most of the targets were found to exhibit molecular function with binding activity and majority were transcription factors, followed by metabolic enzymes, membrane proteins and signaling molecules. The transcription factors targeted by the candidate miRNAs include, TPR like super family proteins, MYB, bHLH, GRF1, NAC, GATA, AP2, Homeo boxleucine zipper, NBS-LRR disease resistant protein. Stress specific expression of miRNAs belonging to miR156, miR162, miR169,miR172, miR393, miR396, miR395, miR398, miR408 family were studied by high-throughput sequencing at whole genome level in several plants such as, Arabidopsis [20, 21], Rice [22], Tomato [23], Wheat [10], Potato [24], Medicago trancatula [25, 26], and Cotton [27]. In this study, we established a set of putative conserved miRNAs in French bean responding to high temperature. Many studies reported miRNAs associated with high temperature stress in other plants including wheat [28], Arabidopsis [29], Brassica [12], Barley [13], Rice [30], Celery [31] etc., however, no reports are found with respect to miRNA expression under high temperature stress in French bean.

Table 2 Targets genes identified for conserved miRNAs in French bean

\begin{tabular}{|c|c|c|}
\hline miRNA & GO number & Description \\
\hline \multirow[t]{7}{*}{ Pvu seq3 } & GO:0004781,GO:0000103 & ATP sulfurylase 1 \\
\hline & GO:0003676,GO:0008270 & Zinc finger protein 1 \\
\hline & & $\alpha$-crystallin domain 32.1 \\
\hline & $\begin{array}{l}\text { GO:0008762,GO:0016491,GO:0050660,GO:0055114, } \\
\text { GO:0003885,GO:0016020 }\end{array}$ & D-arabinono-1,4-lactone oxidase family protein \\
\hline & & Tetratricopeptide repeat (TPR)-like superfamily protein \\
\hline & GO:0008152 & Aconitase/3-isopropylmalate dehydratase protein \\
\hline & $\begin{array}{l}\text { GO:0004455,GO:0009082,GO:0055114,GO:0008652, } \\
\text { GO:0016491 }\end{array}$ & ketol-acid reductoisomerase \\
\hline \multirow[t]{3}{*}{ Pvu seq5 } & GO:0003677,GO:0006355 & AP2/B3-like transcriptional factor family protein \\
\hline & & Histone deacetylase 9 \\
\hline & & Putative ATPase \\
\hline \multirow[t]{2}{*}{ Pvu seq6 } & $\begin{array}{l}\text { GO:0004672,GO:0005524,GO:0006468,GO:0003824, } \\
\text { GO:0016773,GO:0009103,GO:0016020 }\end{array}$ & Protein kinase superfamily protein \\
\hline & GO:0003779,GO:0007010,GO:0015629,GO:0030036 & Profilin 5 \\
\hline Pvu seq7 & GO:0005507,GO:0009055 & Blue-copper-binding protein \\
\hline \multirow[t]{5}{*}{ Pvu seq8 } & GO:0004672,GO:0005524,GO:0006468 & Protein kinase superfamily protein \\
\hline & GO:0008270,GO:0047134,GO:0055114 & Cysteine/Histidine-rich C1 domain family protein \\
\hline & & Late embryogenesis abundant (LEA) hydroxyproline-rich glycoprotein family \\
\hline & $\begin{array}{l}\text { GO:0005506,GO:0009055,GO:0016705,GO:0020037, } \\
\text { GO:0055114 }\end{array}$ & Cytochrome P450, family 710, subfamily A, polypeptide 1 \\
\hline & GO:0003700,GO:0043565,GO:0006355 & WRKY DNA-binding protein 32 \\
\hline \multirow[t]{2}{*}{ Pvu seq9 } & GO:0016758,GO:0008152 & UDP-glucosyl transferase $73 \mathrm{C} 2$ \\
\hline & GO:0005515 & Transducin family protein / WD-40 repeat family protein \\
\hline \multirow[t]{4}{*}{ Pvu seq10 } & GO:0003677 & Myb domain protein 20 \\
\hline & & $\mathrm{C} 2 \mathrm{H} 2$ and $\mathrm{C} 2 \mathrm{HC}$ zinc fingers superfamily protein \\
\hline & $\begin{array}{l}\text { GO:0005515,GO:0005247,GO:0006821,GO:0055085, } \\
\text { GO:0016020 }\end{array}$ & Chloride channel C \\
\hline & GO:0005515 & Disease resistance family protein / LRR family protein \\
\hline \multirow[t]{3}{*}{ Pvu seq13 } & GO:0004185,GO:0006508 & Serine carboxypeptidase-like 31 \\
\hline & & ATP-dependent helicase family protein \\
\hline & GO:0003676 & RNA-binding (RRM/RBD/RNP motifs) family protein \\
\hline \multirow[t]{3}{*}{ Pvu seq14 } & GO:0045454 & PDI-like 1-6 \\
\hline & GO:0005515,GO:0006886,GO:0016192,GO:0030131 & Clathrin adaptor complexes medium subunit family protein \\
\hline & $\begin{array}{l}\text { GO:0003677,GO:0005524,GO:0003676,GO:0008026, } \\
\text { GO:0004386,GO:0008270,GO:0016818 }\end{array}$ & DNA/RNA helicase protein \\
\hline \multirow[t]{3}{*}{ Pvu seq15 } & & NEP-interacting protein 2 \\
\hline & & RING/U-box superfamily protein \\
\hline & GO:0004672,GO:0005524,GO:0006468,GO:0005515 & Leucine-rich repeat protein kinase family protein \\
\hline Pvu seq16 & GO:0016415,GO:0009107,GO:0005737 & Lipoyltransferase 2 \\
\hline \multirow[t]{2}{*}{ Pvu seq17 } & GO:0016779,GO:0009058,GO:0009435 & Nicotinate/nicotinamide mononucleotide adenyltransferase \\
\hline & & ARM repeat superfamily protein \\
\hline
\end{tabular}




\begin{tabular}{|c|c|c|}
\hline \multirow[t]{3}{*}{ Pvu seq18 } & GO:0072488,GO:0016020,GO:0008519 & Ammonium transporter 2 \\
\hline & GO:0016747 & Hydroxycinnamoyl-CoA shikimate/quinate hydroxycinnamoyl transferase \\
\hline & $\begin{array}{l}\text { GO:0004672,GO:0005524,GO:0006468,GO:0048544, } \\
\text { GO:0005529 }\end{array}$ & Protein kinase superfamily protein \\
\hline \multirow[t]{4}{*}{ Pvu seq19 } & GO:0006355,GO:0005634,GO:0003677,GO:0009725 & Auxin response factor 8 \\
\hline & GO:0006355,GO:0005634,GO:0003677,GO:0009725 & Auxin response factor 6 \\
\hline & & Ribosomal protein L7Ae/L30e/S12e/Gadd45 family protein \\
\hline & GO:0016747 & Hydroxycinnamoyl-CoA shikimate/quinate hydroxycinnamoyl transferase \\
\hline \multirow[t]{3}{*}{ Pvu seq20 } & GO:0004672,GO:0005524,GO:0006468 & Protein kinase family protein \\
\hline & GO:0004553,GO:0005975 & O-Glycosyl hydrolases family 17 protein \\
\hline & GO:0005515 & Stress-inducible protein, putative \\
\hline \multirow[t]{3}{*}{ Pvu seq21 } & GO:0043531,GO:0006915,GO:0005524 & LRR and NB-ARC domains-containing disease resistance protein \\
\hline & $\begin{array}{l}\text { GO:0043531,GO:0005515,GO:0007165,GO:0005622, } \\
\text { GO:0045087,GO:0031224,GO:0004888,GO:0006915, } \\
\text { GO:0005524 }\end{array}$ & Disease resistance protein (TIR-NBS-LRR class), putative \\
\hline & $\begin{array}{l}\text { GO:0005515,GO:0043531,GO:0007165,GO:0005622, } \\
\text { GO:0005524,GO:0045087,GO:0031224,GO:0004888, } \\
\text { GO:0006915 }\end{array}$ & Transmembrane receptors;ATP binding \\
\hline Pvu seq22 & & GRAS family transcription factor \\
\hline \multirow[t]{30}{*}{ Pvu seq23 } & GO:0008152 & Pyridoxal phosphate (PLP)-dependent transferases superfamily protein \\
\hline & $\begin{array}{l}\text { GO:0043531,GO:0005515,GO:0007165,GO:0005622, } \\
\text { GO:0045087,GO:0031224,GO:0004888,GO:0006915, } \\
\text { GO:0005524 }\end{array}$ & Disease resistance protein (TIR-NBS-LRR class) family \\
\hline & GO:0004672,GO:0005524,GO:0006468,GO:0005515 & PEP1 receptor 1 \\
\hline & GO:0004672,GO:0005524,GO:0006468,GO:0005515 & Leucine-rich repeat transmembrane protein kinase family protein \\
\hline & GO:0003700,GO:0043565,GO:0046983,GO:0006355 & bZIP transcription factor family protein \\
\hline & GO:0003700,GO:0006355 & Integrase-type DNA-binding superfamily protein \\
\hline & & RING/U-box superfamily protein \\
\hline & GO:0004190,GO:0006508 & Eukaryotic aspartyl protease family protein \\
\hline & GO:0030599,GO:0042545,GO:0005618,GO:0004857 & Plant invertase/pectin methylesterase inhibitor superfamily \\
\hline & GO:0016020,GO:0009765 & Chlorophyll A/B binding protein 1 \\
\hline & GO:0008168,GO:0008152 & S-adenosyl-L-methionine-dependent methyltransferases superfamily protein \\
\hline & & Oxidative stress 3 \\
\hline & $\begin{array}{l}\text { GO:0003700,GO:0043565,GO:0006355,GO:0003677, } \\
\text { GO:0005634 }\end{array}$ & Homeobox-leucine zipper protein family \\
\hline & GO:0003700,GO:0008270,GO:0043565,GO:0006355 & GATA transcription factor 9 \\
\hline & GO:0003697 & Primosome PriB/single-strand DNA-binding \\
\hline & & Pentatricopeptide repeat (PPR) superfamily protein \\
\hline & GO:0003700,GO:0043565,GO:0006355 & WRKY family transcription factor \\
\hline & & Late embryogenesis abundant (LEA) hydroxyproline-rich glycoprotein family \\
\hline & GO:0016788,GO:0006629 & GDSL-like Lipase/Acylhydrolase superfamily protein \\
\hline & GO:0003676 & RNA-binding (RRM/RBD/RNP motifs) family protein \\
\hline & GO:0005524,GO:0016887,GO:0016020 & ABC-2 type transporter family protein \\
\hline & $\begin{array}{l}\text { GO:0004129,GO:0005507,GO:0016020,GO:0009055, } \\
\text { GO:0022900,GO:0016021 }\end{array}$ & Cytochrome oxidase 2 \\
\hline & GO:0003700,GO:0043565,GO:0006355 & WRKY DNA-binding protein 75 \\
\hline & GO:0030528,GO:0006355 & bHLH DNA-binding superfamily protein \\
\hline & $\begin{array}{l}\text { GO:0005506,GO:0009055,GO:0016705,GO:0020037, } \\
\text { GO:0055114 }\end{array}$ & Cytochrome P450, family 71, subfamily B, polypeptide 34 \\
\hline & $\begin{array}{l}\text { GO:0000166,GO:0046872,GO:0003824,GO:0008152, } \\
\text { GO:0016020,GO:0015662,GO:0006812,GO:0006754 }\end{array}$ & $\begin{array}{l}\text { ATPase E1-E2 type family protein / haloacid dehalogenase-like hydrolase family } \\
\text { protein }\end{array}$ \\
\hline & GO:0008138,GO:0006470 & Mitogen-activated protein kinase phosphatase 1 \\
\hline & GO:0031072 & DNAJ heat shock N-terminal domain-containing protein \\
\hline & $\begin{array}{l}\text { GO:0005515,GO:0005524,GO:0016818,GO:0006355, } \\
\text { GO:0005634 }\end{array}$ & Growth-regulating factor 1 \\
\hline & GO:0003700,GO:0043565,GO:0006355,GO:0005634 & Heat shock transcription factor $\mathrm{A} 2$ \\
\hline \multirow[t]{3}{*}{ Pvu seq26 } & & Transmembrane protein-related \\
\hline & GO:0003677,GO:0006306 & Methyltransferase 1 \\
\hline & GO:0003677 & Myb domain protein 21 \\
\hline Pvu seq27 & & Cyclin related \\
\hline Pvu seq28 & GO:0055085,GO:0016021 & Nodulin-like / Major Facilitator Superfamily protein \\
\hline \multirow[t]{4}{*}{ Pvu seq29 } & GO:0003700,GO:0006355 & related to AP2.7 \\
\hline & GO:0003700,GO:0006355 & Integrase-type DNA-binding superfamily protein \\
\hline & GO:0003700,GO:0043565,GO:0006355 & $\begin{array}{l}\text { Homeobox-leucine zipper family protein / lipid-binding START domain- } \\
\text { containing protein }\end{array}$ \\
\hline & GO:0004672,GO:0005524,GO:0006468 & Casein kinase alpha 1 \\
\hline
\end{tabular}




\begin{tabular}{|c|c|c|}
\hline \multirow[t]{7}{*}{ Pvu seq31 } & $\begin{array}{l}\text { GO:0005515,GO:0007165,GO:0005622,GO:0045087, } \\
\text { GO:0031224,GO:0004888 }\end{array}$ & Disease resistance protein (TIR-NBS-LRR class) family \\
\hline & GO:0003700,GO:0006355 & Related to AP2.7 \\
\hline & & Pentatricopeptide (PPR) repeat-containing protein \\
\hline & GO:0016491,GO:0016706,GO:0055114 & Gibberellin 3 -oxidase 1 \\
\hline & & HEAT/U-box domain-containing protein \\
\hline & GO:0005515 & Syntaxin of plants 71 \\
\hline & GO:0005215,GO:0006810,GO:0055085,GO:0016021 & Sulfate transporter 91 \\
\hline \multirow[t]{2}{*}{ Pvu seq32 } & GO:0022857,GO:0055085,GO:0016021 & Major facilitator superfamily protein \\
\hline & $\begin{array}{l}\text { GO:0005524,GO:0016887,GO:0042626,GO:0006810, } \\
\text { GO:0055085,GO:0016021 }\end{array}$ & Multidrug resistance-associated protein 10 \\
\hline \multirow[t]{9}{*}{ Pvu seq33 } & GO:0008236,GO:0006508 & Alpha/beta-Hydrolases superfamily protein \\
\hline & GO:0005524,GO:0044267,GO:0051082,GO:0006457 & TCP-1/cpn60 chaperonin family protein \\
\hline & $\begin{array}{l}\text { GO:0005506,GO:0009055,GO:0016705,GO:0020037, } \\
\text { GO:0055114 }\end{array}$ & Cytochrome P450, family 704, subfamily B, polypeptide 1 \\
\hline & GO:0016020 & Nodulin MtN21 /EamA-like transporter family protein \\
\hline & GO:0043531,GO:0005524,GO:0006915 & NB-ARC domain-containing disease resistance protein \\
\hline & GO:0016742,GO:0009058 & Formyltransferase, putative \\
\hline & GO:0004672,GO:0005524,GO:0006468,GO:0005515 & NSP-interacting kinase 1 \\
\hline & $\begin{array}{l}\text { GO:0045454,GO:0016209,GO:0016491,GO:0055114, } \\
\text { GO:0015035,GO:0009055,GO:0006662 }\end{array}$ & Thioredoxin 2 \\
\hline & GO:0004672,GO:0005524,GO:0006468 & NIMA-related kinase 2 \\
\hline \multirow[t]{7}{*}{ Pvu seq34 } & GO:0003700,GO:0006355 & Related to AP2.7 \\
\hline & & Cleavage and polyadenylation specificity factor 30 \\
\hline & & Late embryogenesis abundant protein (LEA) family protein \\
\hline & $\begin{array}{l}\text { GO:0005506,GO:0009055,GO:0016705,GO:0020037, } \\
\text { GO:0055114 }\end{array}$ & cytochrome $\mathrm{P} 450$, family 81 , subfamily D, polypeptide 8 \\
\hline & $\begin{array}{l}\text { GO:0043531,GO:0005515,GO:0007165,GO:0005622, } \\
\text { GO:0045087,GO:0031224,GO:0004888,GO:0006915, } \\
\text { GO:0005524 }\end{array}$ & Disease resistance protein (TIR-NBS-LRR class) family \\
\hline & GO:0005515 & Cystathionine beta-synthase (CBS) family protein \\
\hline & GO:0030528,GO:0006355 & basic helix-loop-helix (bHLH) DNA-binding superfamily protein \\
\hline \multirow[t]{9}{*}{ Pvu seq36 } & & NB-ARC domain-containing disease resistance protein \\
\hline & GO:0004672,GO:0005524,GO:0006468,GO:0005515 & Leucine-rich receptor-like protein kinase family protein \\
\hline & GO:0003700,GO:0043565,GO:0046983,GO:0006355 & ABA-responsive element binding protein 3 \\
\hline & GO:0003676,GO:0005524,GO:0008026,GO:0004386 & DEA(D/H)-box RNA helicase family protein \\
\hline & GO:0016998 & Peptidoglycan-binding LysM domain-containing protein \\
\hline & $\begin{array}{l}\text { GO:0009055,GO:0016491,GO:0055114,GO:0050660, } \\
\text { GO:0008033 }\end{array}$ & FAD-dependent oxidoreductase family protein \\
\hline & GO:0003700,GO:0043565,GO:0006355,GO:0005634 & Heat shock transcription factor B4 \\
\hline & GO:0005215,GO:0006810,GO:0055085,GO:0016020 & Xanthine/uracil permease family protein \\
\hline & GO:0008168 & S-adenosyl-L-methionine-dependent methyltransferases superfamily protein \\
\hline Pvu seq 37 & GO:0008270 & GATA type zinc finger transcription factor family protein \\
\hline
\end{tabular}

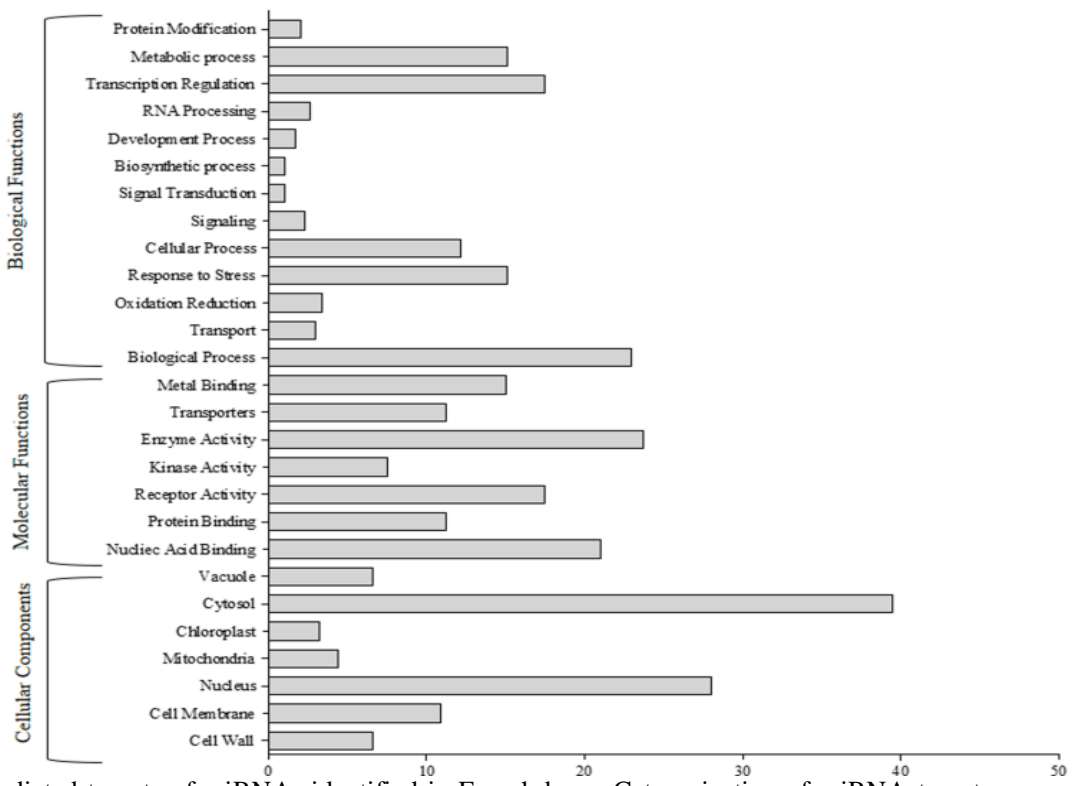

Fig. 3: Gene ontology of the predicted targets of miRNAs identified in French bean. Categorization of miRNA-target genes were performed according to the cellular component, molecular function and biological process. 


\subsection{Expression patterns of conserved and newly identified miRNAs in French bean}

To validate the expression of high temperature responsive miRNAs, we randomly selected eight conserved miRNAs (Pvu seq 3, Pvu seq 10, Pvu seq 13, Pvu seq 16, Pvu seq 20, Pvu seq 21, Pvu seq 27 and Pvu seq 36). Temporal expression of miRNAs were compared with the mock, 10 miRNAs showed differential expressions and could be categorized into three groups: ten fold up regulation was found with Pvu seq 3 while and Pvu seq 16 were up-regulated by 5 fold. Pvu seq 36 and Pvu seq 21 were down regulated by 5 fold. The fold changes were interpreted as logarithm ratio of expression under stress library to the control library. However, Pvu seq 20 and Pvu seq 27 were not affected (Figure 4).

The expression patterns were further verified by Northern blotting and the analogous results were observed. The expression profiles strongly indicate that different miRNA regulation might completely or partially contribute to stress tolerance and differential expression of miRNA is expected to have opposite effect on its target gene. The expression profiles of novel miRNAs are shown in Suppl. 6. The expression patterns of conserved miRNAs were consistent with earlier reports [29, 11]. Through expression abundance of miRNA repertoires many miRNAs were found to have a wide range of expression levels, may be due to miRNA processing and/or stress associated regulation. The up-regulation of the Pvu seq13 and Pvu seq16 appears to play negative role in cell proliferation due to reduced cell division by repressing the target genes GRF and TCP transcription factors respectively. The over expression of miR396 resulted in reduced cell number at the site of cell proliferation and established the interaction of miRNA and GRFs during leaf growth [32]. The qPCR results revealed the expression of target genes is negatively correlated with the expression of their respective candidate miRNAs. Repressions of the targets were found with the up-regulation of their miRNAs and vice versa. We observed the significant fold changes in the expression of miRNAs and their targets. ATP sulpharylase, Serine carboxy peptidase and Lipoyl transferase, were repressed by $2.5,1.0$ and 0.5 fold, while Stress inducible protein, LRR-NBS factor, MYB domain protein, and Heat shock transcription factor B4, were up regulated by 0.2 , 2.0, 3.0, and 3.5 fold respectively. It is observed that, despite of the expression trends of miRNAs, the fold changes were different. However, the target genes exhibited marginal changes in their expression. This may be due to the involvement of transcription regulatory factors other than miRNAs whose expression may not alter due to stress induction (Figure 5). Pvu seq10, Pvu seq21 and Pvu seq36 were observed to be repressed upon high temperature stress by 5 folds. Meanwhile, we also noted the up-regulation of their targets MYB, LRR-NBS and Heat Shock Transcription factor B4, however, the fold change with respect to LRR-NBS was found to be statistically not significant. MYB is a major transcription factor in plants, which play vital roles viz., development, metabolism, hormone signal transduction, disease resistance and environmental cues [33]. OsMYB48 was expressed under PEG, abscisic acid, $\mathrm{H} 2 \mathrm{O} 2$ and dehydration treatments [34]. It is also demonstrated the coordination of 6 MYB factors with SLIM during sulphate assimilation [35]. Mir398 forms the major miRNA studied under high temperature stress. It is shown that down regulation of miR398 in response to oxidative stress would induce the accumulation of its targets genes CSD1, CSD2 and CCS [36]. Guan et al. [29] demonstrated the repression of miR398 in heat exposed Arabidopsis and expression of genes encoding heat stress transcription factors (HSF genes) and heat shock proteins (HSP genes) is reduced in heat-sensitive transgenic plants expressing miR398-resistant forms of CSD1, CSD2 or CCS but is enhanced in the heat-tolerant $\operatorname{csd} 1, \operatorname{csd} 2$ and ccs plants.
A

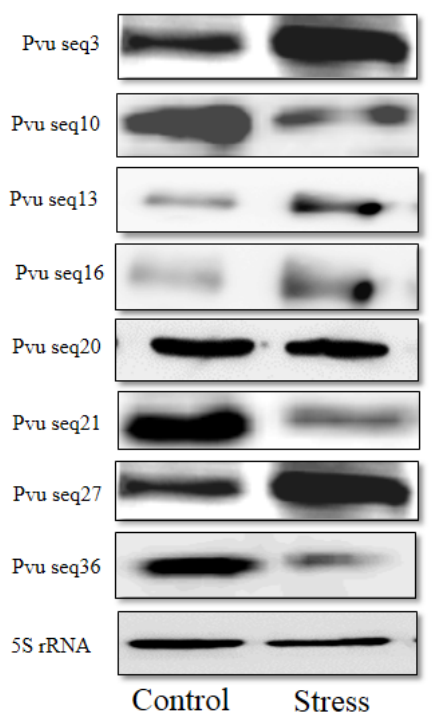

B

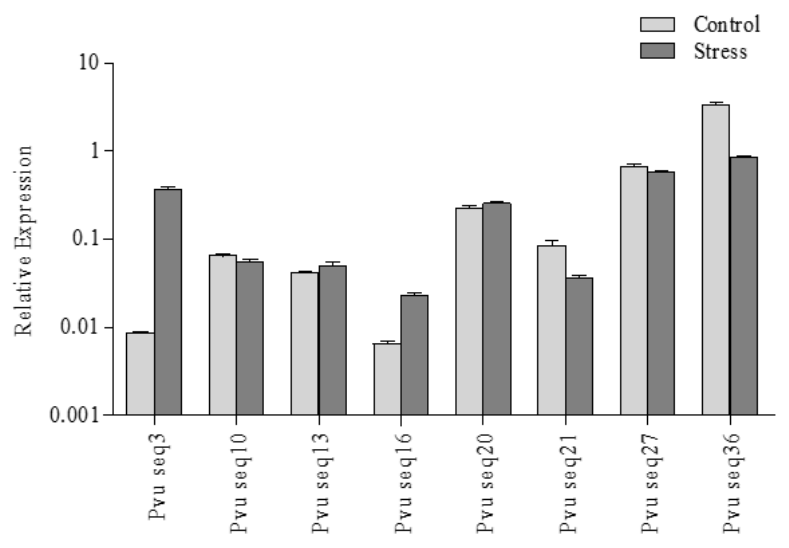

Fig. 4: Expression of miRNAs in French bean. Differentially expressed miRNAs were detected by stem loop qRT-PCR and validated by Northern analysis. Six day-old seedlings (two true leaves) were exposed to high temperature stress at $48{ }^{\circ} \mathrm{C}$ for $6 \mathrm{~h}$, as described in methods. Total RNA from each treatment was extracted, pooled, and determined by RNA gel blotting. A) Northern blot of candidate miRNAs B) qRT-PCR analysis of conserved mature miRNAs. The standard deviations of the data were obtained from the three independent experiments with student's $t$-test ( $\mathrm{p} \leq 0.05)$ using statistics software Graphpad Prism v.5 


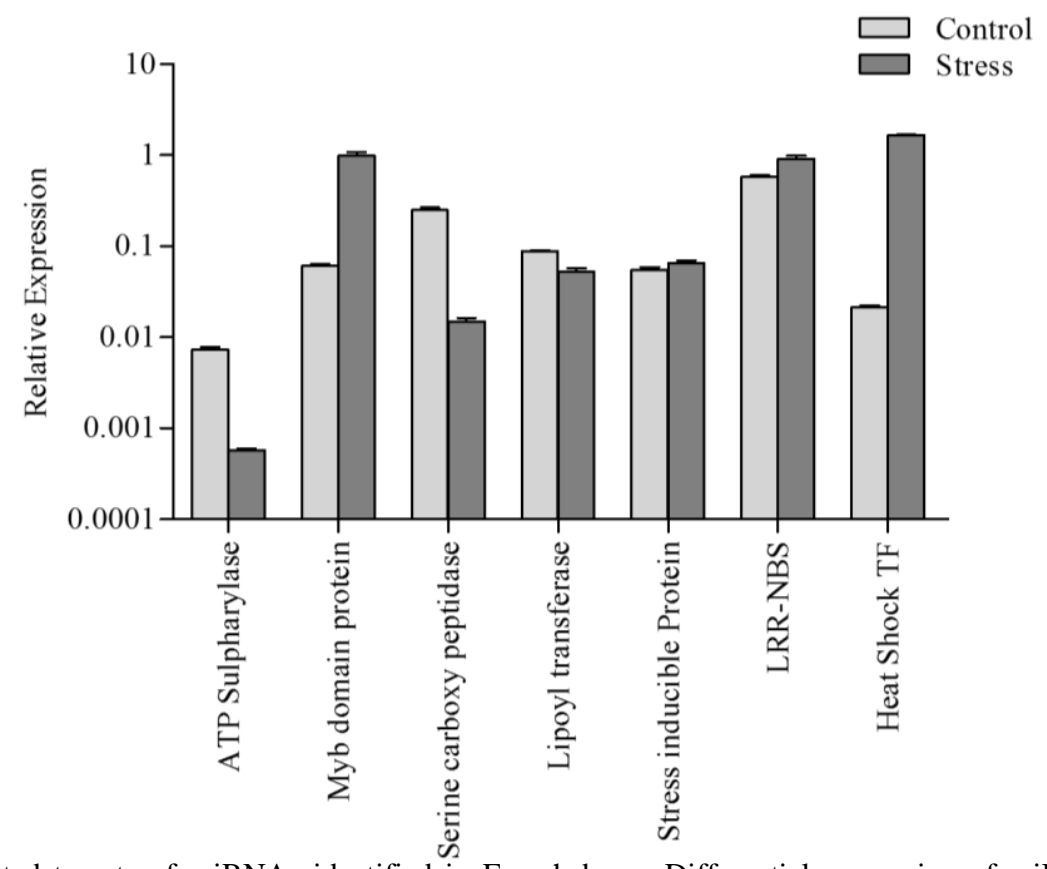

Fig. 5: Validation of predicted targets of miRNAs identified in French bean. Differential expression of miRNA targets were confirmed through qRT-PCR. The standard deviations of the data were obtained from the three independent experiments with student's $t$-test ( $\leq \leq 0.05$ ) using statistics software Graphpad Prism v.5

Suppl. 5: Description of genes targeted by high temperature responsive miRNAs.

\begin{tabular}{|c|c|c|}
\hline miRNA ID. & Target_Acc. & Target Annotation \\
\hline Pvseq3 & Phvul.007G062900.1|PACid:27161269 & ATP sulfurylase 1 \\
\hline Pvseq3 & Phvul.007G118500.1|PACid:27161275 & zinc finger protein 1 \\
\hline Pvseq3 & Phvul.008G264700.1|PACid:27153124 & alpha-crystallin domain 32.1 \\
\hline Pvseq3 & Phvul.001G074300.1|PACid:27163090 & D-arabinono-1,4-lactone oxidase family protein \\
\hline Pvseq3 & Phvul.001G095200.1|PACid:27163727 & Tetratricopeptide repeat (TPR)-like superfamily protein \\
\hline Pvseq5 & Phvul.001G010200.1|PACid:27162704 & Unannotated \\
\hline Pvseq6 & Phvul.002G086900.1|PACid:27169951 & blue-copper-binding protein \\
\hline Pvseq6 & Phvul.002G132600.1|PACid:27170126 & Unannotated \\
\hline Pvseq6 & Phvul.007G249400.1|PACid:27159595 & Protein kinase superfamily protein \\
\hline Pvseq6 & Phvul.007G249400.2|PACid:27159596 & Protein kinase superfamily protein \\
\hline Pvseq6 & Phvul.010G005700.1|PACid:27140434 & Protein of unknown function (DUF3527) \\
\hline Pvseq6 & Phvul.010G005700.2|PACid:27140436 & Protein of unknown function (DUF3527) \\
\hline Pvseq7 & Phvul.002G086900.1|PACid:27169951 & blue-copper-binding protein \\
\hline Pvseq9 & Phvul.001G182500.1|PACid:27162031 & UDP-glucosyl transferase $73 \mathrm{C} 2$ \\
\hline Pvseq9 & Phvul.006G059700.1|PACid:27166626 & Domain of unknown function (DUF2431) \\
\hline Pvseq9 & Phvul.004G028400.1|PACid:27156596 & Protein of unknown function (DUF1666) \\
\hline Pvseq9 & Phvul.011G010400.1|PACid:27152977 & transducin family protein / WD-40 repeat family protein \\
\hline Pvseq9 & Phvul.006G192000.1|PACid:27165602 & FBD, F-box and Leucine Rich Repeat domains containing protein \\
\hline Pvseq10 & Phvul.008G236500.1|PACid:27155183 & myb domain protein 20 \\
\hline Pvseq10 & Phvul.009G089000.1|PACid:27146943 & $\mathrm{C} 2 \mathrm{H} 2$ and $\mathrm{C} 2 \mathrm{HC}$ zinc fingers superfamily protein \\
\hline Pvseq13 & Phvul.006G189100.1|PACid:27166985 & serine carboxypeptidase-like 31 \\
\hline Pvseq13 & Phvul.008G253400.1|PACid:27153972 & Eukaryotic aspartyl protease family protein \\
\hline Pvseq13 & Phvul.005G133500.1|PACid:27149640 & ATP-dependent helicase family protein \\
\hline Pvseq13 & Phvul.005G133500.2|PACid:27149641 & ATP-dependent helicase family protein \\
\hline Pvseq13 & Phvul.006G150400.1|PACid:27165494 & RNA-binding (RRM/RBD/RNP motifs) family protein \\
\hline Pvseq14 & Phvul.011G179700.2|PACid:27152902 & $\mathrm{Ca}(2)$-dependent phospholipid-binding protein (Copine) family \\
\hline Pvseq14 & Phvul.011G179700.1|PACid:27152903 & $\mathrm{Ca}(2)$-dependent phospholipid-binding protein (Copine) family \\
\hline Pvseq14 & Phvul.007G203200.1|PACid:27160187 & Clathrin adaptor complexes medium subunit family protein \\
\hline Pvseq14 & Phvul.010G049600.2|PACid:27141248 & glycine-tRNA ligases \\
\hline Pvseq14 & Phvul.010G049600.1|PACid:27141247 & glycine-tRNA ligases \\
\hline Pvseq14 & Phvul.005G168200.1|PACid:27148705 & PDI-like 1-6 \\
\hline Pvseq14 & Phvul.005G128000.1|PACid:27149260 & DNA/RNA helicase protein \\
\hline Pvseq15 & Phvul.008G252000.1|PACid:27154830 & Unannotated \\
\hline Pvseq15 & Phvul.007G276100.1|PACid:27159665 & Unannotated \\
\hline
\end{tabular}




\begin{tabular}{|c|c|c|}
\hline Pvseq15 & Phvul.007G276100.2|PACid:27159664 & Unannotated \\
\hline Pvseq15 & Phvul.007G275900.1|PACid:27161179 & Unannotated \\
\hline Pvseq15 & Phvul.003G267100.2|PACid:27142387 & NEP-interacting protein 2 \\
\hline Pvseq15 & Phvul.003G267100.1|PACid:27142386 & NEP-interacting protein 2 \\
\hline Pvseq15 & Phvul.007G276700.1|PACid:27159477 & Unannotated \\
\hline Pvseq15 & Phvul.009G133400.1|PACid:27147127 & Unannotated \\
\hline Pvseq15 & Phvul.007G277100.1|PACid:27159851 & Unannotated \\
\hline Pvseq15 & Phvul.007G276200.1|PACid:27161431 & Unannotated \\
\hline Pvseq15 & Phvul.002G105100.1|PACid:27169086 & RING/U-box superfamily protein \\
\hline Pvseq15 & Phvul.007G277000.1|PACid:27160958 & Unannotated \\
\hline Pvseq15 & Phvul.007G276500.1|PACid:27160750 & Unannotated \\
\hline Pvseq15 & Phvul.007G276400.2|PACid:27161554 & Unannotated \\
\hline Pvseq15 & Phvul.007G276400.1|PACid:27161553 & Unannotated \\
\hline Pvseq15 & Phvul.007G276000.1|PACid:27158898 & Unannotated \\
\hline Pvseq15 & Phvul.002G060200.1|PACid:27167520 & Leucine-rich repeat protein kinase family protein \\
\hline Pvseq17 & Phvul.008G279200.1|PACid:27153060 & nicotinate/nicotinamide mononucleotide adenyltransferase \\
\hline Pvseq17 & Phvul.004G026300.2|PACid:27157421 & ARM repeat superfamily protein \\
\hline Pvseq17 & Phvul.004G026300.1|PACid:27157420 & ARM repeat superfamily protein \\
\hline Pvseq17 & Phvul.004G026300.3|PACid:27157419 & ARM repeat superfamily protein \\
\hline Pvseq18 & Phvul.003G267400.1|PACid:27142383 & ammonium transporter 2 \\
\hline Pvseq18 & Phvul.005G183200.1|PACid:27149455 & hydroxycinnamoyl-CoA shikimate/quinate hydroxycinnamoyl transferase \\
\hline Pvseq18 & Phvul.007G077300.1|PACid:27161606 & Protein kinase superfamily protein \\
\hline Pvseq19 & Phvul.008G242400.1|PACid:27154534 & auxin response factor 8 \\
\hline Pvseq19 & Phvul.006G084200.2|PACid:27166910 & auxin response factor 8 \\
\hline Pvseq19 & Phvul.006G084200.1|PACid:27166909 & auxin response factor 8 \\
\hline Pvseq19 & Phvul.002G233600.1|PACid:27167802 & auxin response factor 8 \\
\hline Pvseq19 & Phvul.002G177600.1|PACid:27168787 & auxin response factor 8 \\
\hline Pvseq19 & Phvul.008G197600.1|PACid:27156308 & auxin response factor 6 \\
\hline Pvseq19 & Phvul.005G141300.1|PACid:27149358 & Ribosomal protein L7Ae/L30e/S12e/Gadd45 family protein \\
\hline Pvseq19 & Phvul.005G183200.1|PACid:27149455 & hydroxycinnamoyl-CoA shikimate/quinate hydroxycinnamoyl transferase \\
\hline Pvseq19 & Phvul.007G077300.1|PACid:27161606 & Protein kinase superfamily protein \\
\hline Pvseq19 & Phvul.003G124900.1|PACid:27142544 & Alkaline-phosphatase-like family protein \\
\hline Pvseq19 & Phvul.003G122400.1|PACid:27143589 & P-glycoprotein 11 \\
\hline Pvseq20 & Phvul.009G125200.1|PACid:27146257 & Plant protein of unknown function (DUF869) \\
\hline Pvseq20 & Phvul.009G125200.2|PACid:27146258 & Plant protein of unknown function (DUF869) \\
\hline Pvseq20 & Phvul.008G137900.1|PACid:27156262 & \\
\hline Pvseq20 & Phvul.003G256300.1|PACid:27142161 & S-adenosyl-L-methionine-dependent methyltransferases superfamily protein \\
\hline Pvseq20 & Phvul.003G256100.1|PACid:27142677 & S-adenosyl-L-methionine-dependent methyltransferases superfamily protein \\
\hline Pvseq20 & Phvul.004G127100.2|PACid:27157223 & Microtubule associated protein (MAP65/ASE1) family protein \\
\hline Pvseq20 & Phvul.004G127100.1|PACid:27157222 & Microtubule associated protein (MAP65/ASE1) family protein \\
\hline Pvseq20 & Phvul.002G060800.2|PACid:27170426 & stress-inducible protein, putative \\
\hline Pvseq20 & Phvul.002G060800.1|PACid:27170425 & stress-inducible protein, putative \\
\hline Pvseq21 & Phvul.011G201000.1|PACid:27152390 & LRR and NB-ARC domains-containing disease resistance protein \\
\hline Pvseq21 & Phvul.011G203000.1|PACid:27151641 & NB-ARC domain-containing disease resistance protein \\
\hline Pvseq21 & Phvul.010G004700.1|PACid:27140917 & LRR and NB-ARC domains-containing disease resistance protein \\
\hline Pvseq21 & Phvul.011G181500.1|PACid:27152520 & NB-ARC domain-containing disease resistance protein \\
\hline Pvseq21 & Phvul.004G036300.1|PACid:27158025 & NB-ARC domain-containing disease resistance protein \\
\hline Pvseq21 & Phvul.004G036300.2|PACid:27158024 & NB-ARC domain-containing disease resistance protein \\
\hline Pvseq21 & Phvul.010G136700.1|PACid:27140167 & disease resistance protein (TIR-NBS-LRR class), putative \\
\hline Pvseq21 & Phvul.010G025000.1|PACid:27140443 & Disease resistance protein (TIR-NBS-LRR class) family \\
\hline Pvseq21 & Phvul.002G129200.1|PACid:27168586 & LRR and NB-ARC domains-containing disease resistance protein \\
\hline Pvseq21 & Phvul.002G131100.1|PACid:27169705 & LRR and NB-ARC domains-containing disease resistance protein \\
\hline Pvseq21 & Phvul.002G131200.2|PACid:27171328 & LRR and NB-ARC domains-containing disease resistance protein \\
\hline Pvseq21 & Phvul.002G131200.1|PACid:27171329 & LRR and NB-ARC domains-containing disease resistance protein \\
\hline Pvseq22 & Phvul.010G072700.1|PACid:27140442 & GRAS family transcription factor \\
\hline Pvseq22 & Phvul.010G072900.1|PACid:27141042 & GRAS family transcription factor \\
\hline Pvseq22 & Phvul.008G143400.1|PACid:27153384 & GRAS family transcription factor \\
\hline Pvseq22 & Phvul.008G143400.2|PACid:27153383 & GRAS family transcription factor \\
\hline Pvseq26 & Phvul.010G100800.1|PACid:27140279 & transmembrane protein-related \\
\hline Pvseq27 & Phvul.010G096500.1|PACid:27141429 & Arabidopsis thaliana protein of unknown function (DUF821) \\
\hline Pvseq27 & Phvul.007G045300.1|PACid:27160020 & cyclin-related \\
\hline Pvseq28 & Phvul.002G087600.1|PACid:27168324 & Nodulin-like / Major Facilitator Superfamily protein \\
\hline
\end{tabular}




\begin{tabular}{|c|c|c|}
\hline Pvseq29 & Phvul.005G138300.2|PACid:27148711 & related to $\mathrm{AP} 2.7$ \\
\hline Pvseq29 & Phvul.011G071100.2|PACid:27152560 & related to $\mathrm{AP} 2.7$ \\
\hline Pvseq29 & Phvul.005G138300.1|PACid:27148710 & related to $\mathrm{AP} 2.7$ \\
\hline Pvseq29 & Phvul.011G071100.1|PACid:27152559 & related to $\mathrm{AP} 2.7$ \\
\hline Pvseq29 & Phvul.001G174400.1|PACid:27163589 & related to $\mathrm{AP} 2.7$ \\
\hline Pvseq29 & Phvul.002G016900.1|PACid:27167533 & Integrase-type DNA-binding superfamily protein \\
\hline Pvseq29 & Phvul.003G241900.1|PACid:27141828 & Integrase-type DNA-binding superfamily protein \\
\hline Pvseq29 & Phvul.007G240200.1|PACid:27161577 & related to AP2.7 \\
\hline Pvseq29 & Phvul.001G233200.2|PACid:27162634 & Unannotated \\
\hline Pvseq29 & Phvul.001G233200.1|PACid:27162633 & Unannotated \\
\hline Pvseq29 & Phvul.011G079800.1|PACid:27152785 & Homeobox-leucine zipper family protein / lipid-binding START domain-containing protein \\
\hline Pvseq29 & Phvul.001G033700.2|PACid:27162998 & AT3G20430.1 \\
\hline Pvseq29 & Phvul.001G033700.1|PACid:27162997 & AT3G20430.1 \\
\hline Pvseq29 & Phvul.009G117500.1|PACid:27145893 & like heterochromatin protein (LHP1) \\
\hline Pvseq29 & Phvul.005G030900.1|PACid:27149167 & S-locus lectin protein kinase family protein \\
\hline Pvseq29 & Phvul.002G012700.1|PACid:27167926 & casein kinase alpha 1 \\
\hline Pvseq29 & Phvul.004G132000.1|PACid:27157216 & Peroxidase superfamily protein \\
\hline Pvseq31 & Phvul.010G152200.1|PACid:27141178 & gibberellin 3-oxidase 1 \\
\hline Pvseq31 & Phvul.002G197200.1|PACid:27170332 & O-acetyltransferase family protein \\
\hline Pvseq31 & Phvul.002G004400.1|PACid:27167872 & pentatricopeptide (PPR) repeat-containing protein \\
\hline Pvseq31 & Phvul.008G250900.1|PACid:27153923 & HEAT/U-box domain-containing protein \\
\hline Pvseq31 & Phvul.007G221500.1|PACid:27161668 & syntaxin of plants 71 \\
\hline Pvseq31 & Phvul.008G153500.1|PACid:27156331 & transporter associated with antigen processing protein 1 \\
\hline Pvseq31 & Phvul.010G132000.1|PACid:27139847 & Disease resistance protein (TIR-NBS-LRR class) family \\
\hline Pvseq31 & Phvul.009G249300.1|PACid:27145337 & Tetratricopeptide repeat (TPR)-like superfamily protein \\
\hline Pvseq31 & Phvul.002G095200.1|PACid:27169074 & sulfate transporter 91 \\
\hline Pvseq31 & Phvul.010G084500.2|PACid:27140589 & RNA 2l'-phosphotransferase, Tpt1 / KptA family \\
\hline Pvseq31 & Phvul.010G084500.1|PACid:27140590 & RNA 2l'-phosphotransferase, Tpt1 / KptA family \\
\hline Pvseq33 & Phvul.003G252500.1|PACid:27143606 & Major facilitator superfamily protein \\
\hline Pvseq33 & Phvul.009G165200.1|PACid:27146040 & Unannotated \\
\hline Pvseq36 & Phvul.011G201200.1|PACid:27152203 & retinoblastoma-related 1 \\
\hline Pvseq36 & Phvul.011G201200.2|PACid:27152204 & retinoblastoma-related 1 \\
\hline Pvseq36 & Phvul.008G267500.1|PACid:27154019 & DEA(D/H)-box RNA helicase family protein \\
\hline Pvseq36 & Phvul.002G146700.1|PACid:27170419 & Xanthine/uracil permease family protein \\
\hline Pvseq36 & Phvul.008G244000.1|PACid:27155417 & RNA-binding KH domain-containing protein \\
\hline Pvseq36 & Phvul.009G138500.1|PACid:27147122 & Leucine-rich receptor-like protein kinase family protein \\
\hline Pvseq36 & Phvul.006G016600.1|PACid:27165672 & AT2G24960.2 \\
\hline Pvseq36 & Phvul.006G016600.2|PACid:27165673 & AT2G24960.2 \\
\hline Pvseq36 & Phvul.004G095400.1|PACid:27157244 & Duplicated homeodomain-like superfamily protein \\
\hline Pvseq36 & Phvul.009G227600.1|PACid:27146507 & O-fucosyltransferase family protein \\
\hline Pvseq36 & Phvul.008G057600.1|PACid:27153747 & S-adenosyl-L-methionine-dependent methyltransferases superfamily protein \\
\hline Pvseq37 & Phvul.009G181200.1|PACid:27146418 & GATA type zinc finger transcription factor family protein \\
\hline
\end{tabular}

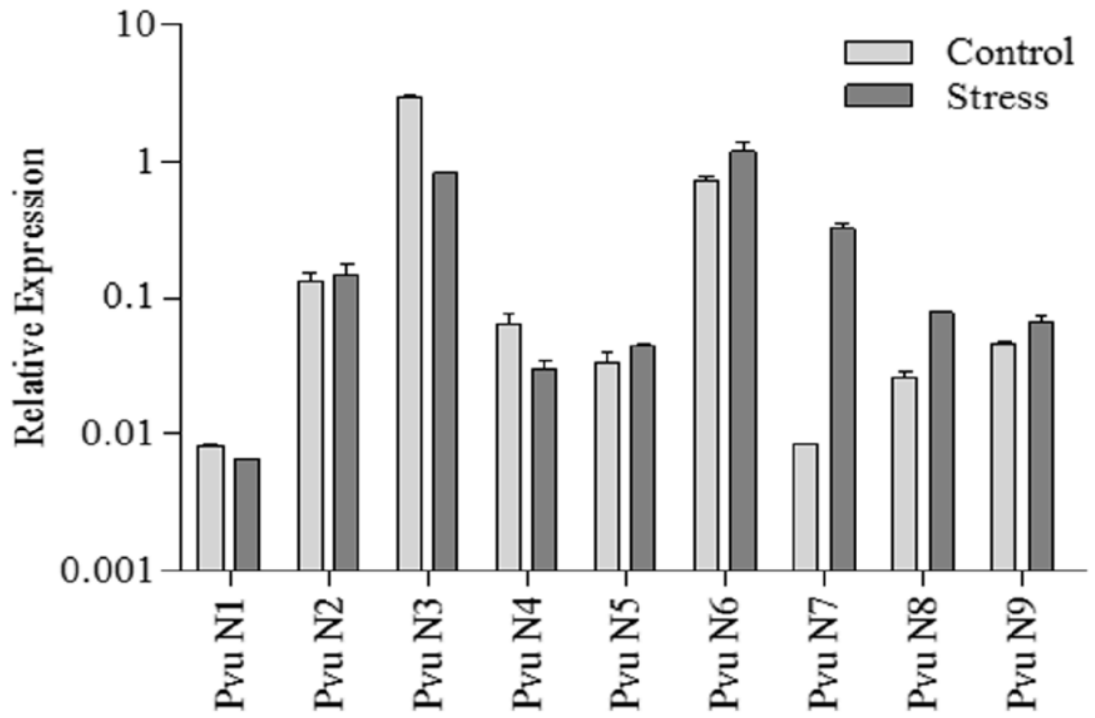

Suppl. 6: Expression assay of novel miRNAs. Expressions were analyzed by RT-qPCR, and normalized against U6 gene. Analysis was carried out in three biological replicates and the student's $t$ test was performed with $p \leq 0.05$ 


\section{CONCLUSION}

Cloning of small RNAs is a starting point to understand their number, diversity and possible roles in different organisms. In this study, we have identified a sum of 36 miRNAs representing 21 families modulated by high temperature and potential targets suggest that miRNAs are directly or indirectly involved in stress response. Validation of these miRNAs emphasis the negative correlation with their target genes. The present work provides important and fairly extensive knowledge about miRNAs and their functions in French bean. Additionally, this knowledge may be useful in studies concerned with methods to increase the yield whilst improving their ability towards stress adaptations.

\section{CONTRIBUTIONS}

Ms. Jyothi has contributed in bringing the data of the following; Construction of cDNA library, characterization of miRNAs and qPCR analysis. Ms. Usha, Ms. Suchithra B and Dr. Sharadamma were involved in computational analysis of miR targets and Northern blot analysis. Dr. Devaraj supported technically for preparing research manuscript. Prof. Rai and Dr. Nagesh Babu are research supervisors/corresponding authors. All the authors have contributed for bringing of this manuscript.

\section{ACKNOWLEDGEMENTS}

Financial support and sponsorship: The research work is supported by University Grants Commission, [No 40-192/2011 (SR)] and Department of Science and Technology, [No SR/FT/LS10/2012] New Delhi, India.

Conflict of Interests: There are no conflicts of interest.

\section{REFERENCES}

1. Hasanuzzaman M, Nahar K, Alam MM, Roychowdhury R, Fujita M. Physiological, biochemical, and molecular mechanisms of heat stress tolerance in plants. Int J Mol Sci 2013; 14:9643-84.

2. Sunkar R, Zhu J. Novel and stress-regulated microRNAs and other small RNAs from Arabidopsis. Plant Cell 2004; 16:2001-2019.

3. Khraiwesh B, Zhua JK, Zhuc J. Role of miRNAs and siRNAs in biotic and abiotic stress responses of plants. Biochemica et Biophysica Acta 2012; 1819:137-148.

4. Kruszka K, Pieczynski M, Windels D, Bielewicz D, Jarmolowski A, Szweykowska-Kulinska Z, Vazquez F. Role of microRNAs and other sRNAs of plants in their changing environments. Journal of Plant Physiology 2012; 169:1664-1672.

5. Ruiz-Ferrer V, Voinnet O. Roles of plant small RNAs in biotic stress responses. Annual Review of Plant Biology 2009; 60:485-510.

6. Katiyar-Agarwal S, Jin H. Role of small RNAs in host-microbe interactions. Annual Review of Phytopathology 2010; 48:225-246.

7. Lv DK, Bai X, Li Y, Ding XD, Ge Y, Cai H, Ji W, Wu N, Zhu YM. Profiling of cold-stress-responsive miRNAs in rice by microarrays. Gene 2010; 459:39-47.

8. Jian X, Zhang L, Li G, Zhang L, Wang X, Cao X, Fang X, Chen F. Identification of novel stress regulated microRNAs from Oryza sativa L. Genomics 2010; 95:47-55.

9. Bartels D, Sunkar R. Drought and salt tolerance in plants. Critical Reviews in Plant Sciences 2005; 24:23-58.
10. Xin M, Wang Y, Yao Y, Xie C, Peng H, Ni Z, Sun Q. Diverse set of microRNAs are responsive to powdery mildew infection and heat stress in wheat (Triticum aestivum L.). BMC Plant Biology 2010; 10:123-36.

11. Yu X, Wang H, Lu Y, de Ruiter M, Cariaso M, Prins M, van Tunen A, He Y. Identification of conserved and novel microRNAs that are responsive to heat stress in Brassica rapa. J Exp Bot 2012; 63:102538.

12. Kruszka K, Pacak A, Swida-Barteczka A, Nuc P, Alaba S, Wroblewska Z, Karlowski W, Jarmolowski A, SzweykowskaKulinska Z. Transcriptionally and post-transcriptionally regulated microRNAs in heat stress response in barley. J Exp Bot $2014 ; 65: 6123-6135$.

13. Arenas-Huertero CB, Rabanal F, Blanco-Melo D, De la Rosa C, Estrada-Navarrete G, Sanchez F, Covarrubias AA, Reyes JL. Conserved and novel miRNAs in the legume Phaseolus vulgaris in response to stress. Plant Mol Biol 2009; 70:385-401.

14. Valdes-Lopez O, Yang SS, Aparicio-Fabre R, Graham PH, Reyes JL, Vance CP, Hernandez G. MicroRNA expression profile in common bean (Phaseolus vulgaris) under nutrient deficiency stresses and manganese toxicity. New Phytol 2010; 187:805-818.

15. Pelaez P, Trejo MS, Iniguez LP, Estrada-Navarrete G, Covarrubias AA, Reyes JL, Sanchez F. Identification and characterization of microRNAs in Phaseolus vulgaris by high-throughput sequencing. BMC Genomics 2012; 13:83

16. Nagesh babu R, Jyothi MN, Sharadamma N, Rai DV, Devaraj VR Expression of miRNAs regulates growth and development of French bean (Phaseolus vulgaris) under Salt and Drought Stress conditions. Int. Res. J. Biol Sci 2013; 2:1-6.

17. Nagesh babu R, Jyothi MN, Usha, Sharadamma N, Rai DV, Devaraj VR. Identification of miRNAs from French bean (Phaseolus vulgaris) under low nitrate stress. Turkish Journal of Biochemistry 2014; 39:1-8.

18. Zuker M. Mfold web server for nucleic acid folding and hybridization prediction. Nucleic Acids Res 2003; 31:3406-3415.

19. Meyers BC, Axtell MJ, Bartel B, Bartel DP, Baulcombe D, Bowman JL, Cao X, Carrington JC, Chen X, Green PJ, Griffiths S, Jacobsen SE, Mallory AC, Martienssen RA, Poethig RS, Qi Y, Vaucheret H, Voinnet O, Watanabe Y, Weigel D, Zhu JK. Criteria for annotation of plant microRNAs. Plant Cell 2008; 20:3186-3190.

20. Tagami Y, Inaba N, Kutsuna N, Kurihara Y, Watanabe Y. Specific enrichment of miRNAs in Arabidopsis thaliana infected with Tobacco mosaic virus. DNA Res 2007; 14:227-233.

21. Liang $\mathrm{G}, \mathrm{He} \mathrm{H}, \mathrm{Yu} \mathrm{D}$. Identification of nitrogen starvationresponsive microRNAs in Arabidopsis thaliana. PLoS One 2012; 7:1-11.

22. Mishra NS, Vikash Kumar, Sopory SK, Mukherjee SK. Cloning and validation of novel miRNA from basmati rice indicates cross talk between abiotic and biotic stresses. Mol Genet Genomics 2009; 282:463-474.

23. Moxon S, Jing R, Szittya G, Schwach F, Rusholme-Pilcher RL, Moulton V, Dalmay T. Deep sequencing of tomato short RNAs identifies microRNAs targeting genes involved in fruit ripening. Genome Res 2008; 18:1602-1609.

24. Zhang N, Yang J, Wang Z, Wen Y, Wang J, He W, Liu B, Si H, Wang D. Identification of novel and conserved microRNAs related to drought stress in Potato by deep sequencing. PLoSOne 2014; 18:1-9.

25. Trindade I, Capitao C, Dalmay T, Fevereiro MP, Santos DM. miR398 and miR408 are up-regulated in response to water deficit in Medicago truncatula. Planta 2010; 231:705-716.

26. Jagadeeswaran G, Zheng Y, Li YF, Shukla LI, Matts J, Hoyt P, Macmil SL, Wiley GB, Roe BA, Zhang W, Sunkar R. Cloning and characterization of small RNAs from Medicago truncatula reveals four novel legume-specific microRNA families. New Phytol 2009; 184:85-98 
27. Khan-Barozai MY, Irfan M, Yousaf R, Ali I, Qaisar U, Maqbool A, Zahoor M, Rashid B, Hussnain T, Riazuddin S. Identification of micro-RNAs in cotton. Plant Physiol Biochem 2008; 46:739- 751.

28. Kumar RR, Pathak H, Sharma SK, Kala YK, Nirjal MK, Singh GP, Goswami S, Rai RD. Novel and conserved heat-responsive microRNAs in wheat (Triticum aestivum L.). Funct Integr Genomics 2014; 10:1-26.

29. Guan Q, Lu X, Zeng H, Zhang Y, Zhu J. Heat stress induction of miR398 triggers a regulatory loop that is critical for thermo-tolerance in Arabidopsis. Plant J 2013; 74:840-51.

30. Mittal D, Madhyastha DA, Grover A. Genome-wide transcriptional profiles during temperature and oxidative stress reveal coordinated expression patterns and overlapping regulons in Rice. PLoS ONE 2012; 7:1-15.

31. Li MY, Wang F, Xu ZS, Jiang Q, Ma J, Tan GF, Xiong AS. High throughput sequencing of two celery varieties small RNAs identifies microRNAs involved in temperature stress response. BMC Genomics 2014; $15: 242$.

32. Debernardi JM, Rodriguez RE, Mecchia MA, Palatnik JF. Functional Specialization of the Plant miR396 Regulatory Network through Distinct MicroRNA-Target Interactions. PLoS Genet 2012; 8:1-14.
33. Pashkovskiy PP, Ryazansky SS. Biogenesis, Evolution, and Functions of Plant microRNAs. Biochemistry (Moscow) 2013; 78:627-637.

34. Xiong H, Li J, Liu P, Duan J, Zhao Y, Guo X, Li Y, Zhang H, Ali J, Li Z. Over expression of OsMYB48-1, a novel MYB-related transcription factor enhances drought and salinity tolerance in Rice. PLoS ONE 2014; 9:1-13.

35. Yatusevich R, Mugford SG, Matthewman C, Gigolashvili T, Frerigmann H, Delaney S, Koprivova A, Flugge UI, Kopriva S. Genes of primary sulfate assimilation are part of the glucosinolate biosynthetic network in Arabidopsis thaliana. Plant J 2010; 62:1-11.

36. Lu X, Guan Q, Zhu J. Down-regulation of CSD2 by a heat-inducible miR398 is required for thermo-tolerance in Arabidopsis. Plant Signaling \& Behavior 2013; 8:1-3.

\section{How to cite this article:}

Jyothi MN, Usha S, Suchithra B, Sharadamma N, Rai DV, Devaraj VR, Nagesh babu R. Identification and profiling of high temperature responsive miRNAs in French bean (Phaseolus vulgaris L). J App Biol Biotech. 2016; 4 (06): 038-058. 